\title{
Equimorphism invariants for scattered linear orderings
}

\author{
by
}

Antonio Montalbán (Chicago, IL)

\begin{abstract}
Two linear orderings are equimorphic if they can be embedded in each other. We define invariants for scattered linear orderings which classify them up to equimorphism. Essentially, these invariants are finite sequences of finite trees with ordinal labels.

Also, for each ordinal $\alpha$, we explicitly describe the finite set of minimal scattered equimorphism types of Hausdorff rank $\alpha$. We compute the invariants of each of these minimal types.
\end{abstract}

1. Introduction. We say that a linear ordering is scattered if the order type of the rationals does not embed in it. People have been interested in this class of linear orderings for a long time. One of the earliest results is the following, first proved by Hausdorff [Hau08], and rediscovered by Erdös and Hajnal [EH63].

THEOREM 1.1 (Hausdorff). Let $\mathbb{S}$ be the smallest class of linear orderings such that

- $1 \in \mathbb{S}$;

- if $\mathcal{A}, \mathcal{B} \in \mathbb{S}$, then $\mathcal{A}+\mathcal{B} \in \mathbb{S}$;

- if $\kappa$ is a regular cardinal and $\left\{\mathcal{A}_{\gamma}: \gamma \in \kappa\right\} \subseteq \mathbb{S}$, then both $\sum_{\gamma \in \kappa} \mathcal{A}_{\gamma}$ and $\sum_{\gamma \in \kappa^{*}} \mathcal{A}_{\gamma}$ belong to $\mathbb{S}$.

Then $\mathbb{S}$ is the class of scattered linear orderings. (The notation used is explained in the background section below.)

Another important contribution of Hausdorff to the study of scattered linear orderings is the definition of the Hausdorff rank (see [Ros82, Chapter 5]). He first defined an operation on linear orderings which is similar to

2000 Mathematics Subject Classification: Primary 03E04.

Key words and phrases: invariant, scattered, embeddability, linear order.

This research is part of my Ph.D. thesis [Mon05a] at Cornell University. I am grateful to my thesis adviser Richard A. Shore for all his help. The research was partially supported by NSF Grant DMS-0100035. 
the Cantor-Bendixson derivative on topological spaces: Given a linear ordering $\mathcal{L}$, let $\mathcal{L}^{\prime}$ be the linear ordering obtained by collapsing the elements which have only finitely many elements between them. Informally, the Hausdorff rank of $\mathcal{L}$ is the least ordinal $\alpha$ such that the $\alpha$ th iterate of this operation on $\mathcal{L}$ is finite. Here is the definition we will use.

Definition 1.2. Given a linear ordering $\mathcal{L}$ and an ordinal $\alpha$, we define an equivalence relation $\approx_{\alpha}$ on $\mathcal{L}$ by transfinite induction as follows. Let $\approx_{0}$ be the identity relation. For $x, y \in \mathcal{L}$, let $x \approx_{\alpha} y$ if and only if for some $\beta<\alpha$, there are only finitely many $\approx_{\beta}$-equivalence classes between $x$ and $y$. Let $\mathcal{L}^{(\alpha)}$ be the linear ordering which consists of the $\approx_{\alpha}$-equivalence classes ordered in the obvious way. We let the Hausdorff rank of $\mathcal{L}, \operatorname{rk}(\mathcal{L})$, be the least ordinal $\alpha$ such that $\mathcal{L}^{(\alpha)}$ is finite. If no such $\alpha$ exists, we let $\operatorname{rk}(\mathcal{L})=\infty$. We will usually omit the word Hausdorff and just refer to the rank of a linear ordering.

Hausdorff proved that a linear ordering is scattered if and only if $\operatorname{rk}(\mathcal{L})$ $\neq \infty$.

The definition above is slightly different from some other definitions of Hausdorff rank found in the literature, but is essentially the same. We prefer it to other definitions because it has the following three properties. Let $\mathcal{A}$ and $\mathcal{B}$ be linear orderings. Then

(1) if $\mathcal{A}$ embeds in $\mathcal{B}$, then $\operatorname{rk}(\mathcal{A}) \leq \operatorname{rk}(\mathcal{B})$;

(2) $\operatorname{rk}(\mathcal{A}+\mathcal{B})=\max (\operatorname{rk}(\mathcal{A}), \operatorname{rk}(\mathcal{B}))$;

(3) $\operatorname{rk}(\mathcal{A} \cdot \mathcal{B})=\operatorname{rk}(\mathcal{A})+\operatorname{rk}(\mathcal{B})$.

After Hausdorff's results, the following important structural result about the class of scattered linear orderings was conjectured by Fraïssé in [Fra48] in the countable case. It was proved by Richard Laver twenty three years later.

THEOREM 1.3 ([Lav71]). The class of scattered linear orderings is wellquasiordered by the relation of embeddability.

A well-quasiordering is a quasiordering which has no infinite descending sequences and no infinite antichains.

Moreover, Laver proved that the class of scattered linear orderings is a better-quasiordering. Better-quasiorderings are a particular case of wellquasiorderings introduced by Nash-Williams in [NW68]. Then, for example, using Nash-Williams' theorem on transfinite sequences [NW68], we deduce that the class of ideals of scattered linear orderings (i.e., downwards closed sets of linear orderings), ordered by inclusion, is also well-quasiordered.

In the proof of Laver's result, indecomposable linear orderings play a very important role. A linear ordering $\mathcal{L}$ is indecomposable if whenever $\mathcal{L}=\mathcal{A}+\mathcal{B}$, either $\mathcal{L}$ embeds in $\mathcal{A}$ or $\mathcal{L}$ embeds in $\mathcal{B}$. Along with the theorem above, Laver 
proved some structural results about the class of $\sigma$-scattered linear orderings (see Definition 5.2). When we restrict these results to the class of scattered linear orderings we obtain the following theorem.

Theorem 1.4 ([Lav71]).

(1) Every scattered linear ordering can be written as a finite sum of indecomposable linear orderings.

(2) Every indecomposable linear ordering is either a $\kappa$-sum or a $\kappa^{*}$-sum of indecomposable linear orderings of smaller rank, where $\kappa$ is some regular cardinal.

When a linear ordering $\mathcal{A}$ can be embedded into another linear ordering $\mathcal{B}$, we write $\mathcal{A} \preccurlyeq \mathcal{B}$. The orderings $\mathcal{A}$ and $\mathcal{B}$ are equimorphic if $\mathcal{A} \preccurlyeq \mathcal{B} \preccurlyeq \mathcal{A}$. We then write $\mathcal{A} \sim \mathcal{B}$. Everything mentioned so far about scattered linear orderings is not really about isomorphism types of linear orderings, but actually about equimorphism types. The properties of being scattered, of being indecomposable, and of having a certain rank are preserved under equimorphisms. Also, the operations of taking finite sums, products and $\kappa$-sums are well defined on equimorphism types.

In this paper we are interested in the structure of equimorphism types of scattered linear orderings. We use Laver's work and assign to each scattered linear ordering $\mathcal{L}$ a finite sequence $\operatorname{Inv}(\mathcal{L})$ of finite trees labeled by ordinals and signs in $\{-,+\}$. This assignment is an equimorphism invariant, that is, given scattered linear orderings $\mathcal{A}$ and $\mathcal{B}$, we have

$$
\mathcal{A} \sim \mathcal{B} \Leftrightarrow \operatorname{Inv}(\mathcal{A})=\operatorname{Inv}(\mathcal{B})
$$

Let $\mathbb{S}$ denote the class of equimorphism types of scattered linear orderings and $\mathbb{H}$ the class of equimorphism types of scattered indecomposable linear orderings. From now on, an indecomposable means a scattered and indecomposable linear ordering, unless otherwise stated. Let $\mathbb{H}_{\alpha}=\{\mathcal{L} \in \mathbb{H}$ : $\operatorname{rk}(\mathcal{L})<\alpha\}$.

Jullien [Jul69, Theorem IV.6.2] proved the following. Let $\mathcal{L}$ be a scattered linear ordering and let $\left\langle\mathcal{A}_{0}, \ldots, \mathcal{A}_{n-1}\right\rangle$ be a sequence of indecomposables such that $\mathcal{L}=\mathcal{A}_{0}+\cdots+\mathcal{A}_{n-1}$ and $n$ is minimum possible. Then $\left\langle\mathcal{A}_{0}, \ldots, \mathcal{A}_{n-1}\right\rangle$ is unique up to equimorphism (see also [Mona, Subsection 3.2]). So, to define $\operatorname{Inv}(\mathcal{L})$, it is enough to define invariants for the class of indecomposable linear orderings. We will assign a finite tree $\mathrm{T}\left(\mathcal{A}_{i}\right)$ to each indecomposable linear ordering and then take

$$
\operatorname{Inv}(\mathcal{L})=\left\langle\mathrm{T}\left(\mathcal{A}_{0}\right), \ldots, \mathrm{T}\left(\mathcal{A}_{n-1}\right)\right\rangle .
$$

A linear ordering is indecomposable to the left (resp. right) if, whenever $\mathcal{A}$ and $\mathcal{B}$ are linear orderings such that $\mathcal{L}=\mathcal{A}+\mathcal{B}$, then $\mathcal{L}$ is equimorphic to $\mathcal{A}$ (resp. to $\mathcal{B}$ ). Another result of Jullien [Jul69, Theorem IV.3.3] is that 
every indecomposable linear ordering is either indecomposable to the right or to the left. (See also [Fra00, 6.3.4(3)] and [Ros82, Lemma 10.3], and see [Monb] for a reverse mathematics analysis of this statement.) Let $\varepsilon_{\mathcal{L}}$ be + if $\mathcal{L}$ is indecomposable to the right, and - if it is indecomposable to the left. Given $\mathcal{L} \in \mathbb{H}$, let $\mathbb{I}_{\mathcal{L}}=\{\mathcal{A} \in \mathbb{H}: 1+\mathcal{A}+1 \prec \mathcal{L}\}$. Note that $\mathbb{I}_{\mathcal{L}} \subseteq \mathbb{H}_{\mathrm{rk}(\mathcal{L})}$ and that $\mathbb{I}_{\mathcal{L}}$ is downwards closed. Subsets of $\mathbb{H}$ which are downwards closed will be called ideals of $\mathbb{H}$. We will prove in Corollary 2.6 that $\mathcal{L}$ is determined by $\varepsilon_{\mathcal{L}}$ and $\mathbb{I}_{\mathcal{L}}$. We use this fact to define $\mathrm{T}(\mathcal{L})$, the invariant of $\mathcal{L}$.

Definition 1.5. We assign a finite tree, $\mathrm{T}(\mathcal{L})$, with labels in $\mathcal{O} n \times$ $\{+,-\}$, to each $\mathcal{L} \in \mathbb{H}$ (where $\mathcal{O} n$ is the class of ordinals). Let $\left\{\mathcal{L}_{1}, \ldots, \mathcal{L}_{k}\right\}$ be the set of minimal elements of $\mathbb{H}_{\mathrm{rk}(\mathcal{L})} \backslash \mathbb{I}_{\mathcal{L}}$. Define

$$
\mathrm{T}(\mathcal{L})=\left[\left\langle\operatorname{rk}(\mathcal{L}), \varepsilon_{\mathcal{L}}\right\rangle ; \mathrm{T}\left(\mathcal{L}_{1}\right), \ldots, \mathrm{T}\left(\mathcal{L}_{k}\right)\right] .
$$

That is, $\mathcal{T}(\mathcal{L})$ is a tree with a root labeled $\left\langle\operatorname{rk}(\mathcal{L}), \varepsilon_{\mathcal{L}}\right\rangle$ and with $k$ branches $\mathrm{T}\left(\mathcal{L}_{1}\right), \ldots, \mathrm{T}\left(\mathcal{L}_{k}\right)$.

The set of minimal elements of $\mathbb{H}_{\operatorname{rk}(\mathcal{L})} \backslash \mathbb{I}_{\mathcal{L}}$ is finite because there are no infinite antichains in $\mathbb{H}$, and determines $\mathbb{I}_{\mathcal{L}}$ because $\mathbb{H}$ is well-founded, and hence for $\mathcal{A} \in \mathbb{H}_{\mathrm{rk}(\mathcal{L})}, \mathcal{A} \in \mathbb{I}_{\mathcal{L}}$ if and only if for no $i \leq k, \mathcal{L}_{i} \preccurlyeq \mathcal{A}$.

The rest of the paper is dedicated to proving that these invariants are actually equimorphism invariants and to showing that they are somewhat constructive. We do the latter by showing that the definition of the embeddability relation on the invariants is relatively simple, and that we can easily characterize the finite trees that correspond to invariants. We also compute the invariants of every linear ordering which is a product of linear orderings of the form $\omega^{\alpha}$ or $\left(\omega^{\alpha}\right)^{*}$.

Let

$$
\mathcal{T} r=\{\mathrm{T}(\mathcal{L}): \mathcal{L} \in \mathbb{H}\} \quad \text { and } \quad \mathcal{I} n=\{\operatorname{Inv}(\mathcal{L}): \mathcal{L} \in \mathbb{S}\} .
$$

In Section 2 we define a relation $\preccurlyeq$ on $\mathcal{I} n$ such that Inv $:\langle\mathbb{S}, \preccurlyeq\rangle \rightarrow\langle\mathcal{I} n, \preccurlyeq\rangle$ is an isomorphism. We define $\preccurlyeq$ in such a way that, given $S, T \in \mathcal{I} n$, we can tell whether $S \preccurlyeq T$ via a finite manipulation of symbols, assuming we can compare the ordinals that appear in the labels of $S$ and $T$ and their cofinalities.

In Proposition 2.16 we characterize the finite sequences of finite trees with labels in $\mathcal{O} n \times\{-,+\}$ which belong to $\mathcal{I} n$. This characterization is based on Proposition 2.14, where we characterize the finite trees with labels in $\mathcal{O} n \times\{-,+\}$ which belong to $\mathcal{T}_{r}$. All the conditions in these characterizations can be checked using a finite algorithm, except 2.14(4), which requires the computation of the cofinality of an ideal. This condition always holds when we deal with countable linear orderings. So, we get a characterization of the elements of $\mathcal{I n}_{\omega_{1}}=\left\{\operatorname{Inv}(\mathcal{L}): \mathcal{L} \in \mathbb{S} \& \operatorname{rk}(\mathcal{L})<\omega_{1}\right\}$ via a finite algorithm. 
To find invariants of linear orderings, it is necessary to find the minimal linear orderings of complements of ideals. The first result in this direction is the following.

TheOREM 1.6 (Hausdorff, see [Ros82]). Let $\kappa$ be a regular cardinal and $\mathcal{L}$ be a scattered linear ordering. Then $\kappa \leq|\mathcal{L}|$ if and only if either $\kappa \preccurlyeq \mathcal{L}$ or $\kappa^{*} \preccurlyeq \mathcal{L}$.

Since a scattered linear ordering has rank $\geq \kappa$ if and only if it has size $\geq \kappa$, it follows that $\left\{\kappa, \kappa^{*}\right\} \subset \mathbb{S}$ is the set of minimal equimorphism types of rank $\kappa$. For each ordinal $\alpha$, since $\mathbb{S}$ is well-quasiordered, there exists a finite set $\mathbb{F}_{\alpha}$ of minimal equimorphism types of rank $\alpha$. In Section 3 , we explicitly define the elements of $\mathbb{F}_{\alpha}$ for each $\alpha$. In Section 4 we find the invariants of these minimal equimorphism types.

As mentioned before, the class of ideals of $\mathbb{H}$, ordered by inclusion, is also a well-quasiordering. In Section 3, for each $\alpha$, we also explicitly define a finite set of ideals of $\mathbb{H}$ of rank $\alpha$ which contains all the minimal ideals of rank $\alpha$. Then, in Section 4, we find the invariants of these ideals. We will use these invariants to describe an algorithm that checks if an ideal of $\mathbb{H}$ has a certain rank, as needed to verify condition 2.14(3).

Many ideas in this paper originated in [Mon05b], where we proved that every hyperarithmetic linear ordering is equimorphic to a computable one, extending an old result of Spector about hyperarithmetic ordinals. The definitions of the invariants and the definition of minimal ideals of a certain rank are essentially given there for the case of countable linear orderings, but this is not explicity stated. Peter Cholak, after a talk the author gave in Notre Dame, suggested that there might be some relation between the work in [Mon05b] and equimorphism invariants. An important tool used in [Mon05b] is the concept of signed trees. Some results that were already proved in [Mon05b] only for countable linear orderings, are proved here for arbitrary cardinality and without using signed trees. Even though the proofs with signed trees are cleaner, we do not know how to generalize the concept of signed trees to arbitrary cardinality preserving their nice properties. At the end of Section 2 we mention how the results in [Mon05b] could be deduced as an application of the results on equimorphism invariants proved here.

In the last section we mention extensions of our results to the class of $\sigma$-scattered linear orderings, and some questions that are left open.

1.1. Background on linear orderings. The reader can find background information about linear orderings in the introductory chapter of [Ros82]. Basic knowledge about ordinals is assumed. The reader can learn about ordinals in any basic textbook in set theory, as for example [Kun80]. Now, we define the notation we will use and we prove some basic lemmas about indecomposable linear orderings. Let $\mathcal{A}=\left\langle A, \leq_{\mathcal{A}}\right\rangle$ be a linear ordering. We 
might sometimes abuse notation and write $x \in \mathcal{A}$ instead of $x \in A$. The reverse linear ordering of $\mathcal{A}$ is $\mathcal{A}^{*}=\left\langle A, \geq_{\mathcal{A}}\right\rangle$. We let $\mathcal{A}^{+}=\mathcal{A}$ and $\mathcal{A}^{-}=\mathcal{A}^{*}$. Let $\mathcal{B}=\left\langle B, \leq_{\mathcal{B}}\right\rangle$ be another linear ordering. The product, $\mathcal{A} \cdot \mathcal{B}$, of $\mathcal{A}$ and $\mathcal{B}$ is obtained by substituting a copy of $\mathcal{A}$ for each element of $\mathcal{B}$. That is, $\mathcal{A} \cdot \mathcal{B}=\left\langle A \times B, \leq_{\mathcal{A} \cdot \mathcal{B}}\right\rangle$ where $\langle x, y\rangle \leq_{\mathcal{A} \cdot \mathcal{B}}\left\langle x^{\prime}, y^{\prime}\right\rangle$ iff either $y<_{\mathcal{B}} y^{\prime}$, or $y=y^{\prime}$ and $x \leq_{\mathcal{A}} x^{\prime}$. The sum, $\sum_{i \in \mathcal{A}} \mathcal{B}_{i}$, of a set $\left\{\mathcal{B}_{i}\right\}_{i \in A}$ of linear orderings indexed by another linear ordering $\mathcal{A}$ is constructed by substituting a copy of $\mathcal{B}_{i}$ for each element $i \in A$. So, for example, $\mathcal{A} \cdot \mathcal{B}=\sum_{i \in \mathcal{B}} \mathcal{A}$. When $\mathcal{A}=\{0<1<\cdots<m-1\}$, we sometimes write $\mathcal{B}_{0}+\cdots+\mathcal{B}_{m-1}$ or $\sum_{i=0}^{m-1} \mathcal{B}_{i}$ instead of $\sum_{i \in \mathcal{A}} \mathcal{B}_{i}$

We use $\omega$ to denote the ordering of the natural numbers, and 1 for the linear ordering with one element.

The powers of $\omega$ are defined as follows: $\omega^{0}=1$ and for an ordinal $\alpha$, $\omega^{\alpha}=\sup \left\{\omega^{\beta} \cdot \omega: \beta<\alpha\right\}$. We write $\omega^{\alpha *}$ for $\left(\omega^{\alpha}\right)^{*}$. Cantor proved that for every ordinal $\alpha$ there exists a finite sequence of ordinals $\alpha_{0} \geq \alpha_{1} \geq \cdots \geq \alpha_{k}$ such that

$$
\alpha=\omega^{\alpha_{0}}+\omega^{\alpha_{1}}+\cdots+\alpha^{\alpha_{k}} .
$$

This decomposition is called the Cantor normal form of $\alpha$. It can be proved by induction that $\operatorname{rk}\left(\omega^{\alpha}\right)=\alpha$. Moreover, $\omega^{\alpha}$ is the least ordinal of rank $\alpha$. It is also known, and not hard to prove, that an ordinal is indecomposable if and only if it is of the form $\omega^{\alpha}$.

Given a set $X$, we denote the set of finite sequences from $X$ by $X^{<\omega}$.

Given a partial ordering $\mathcal{P}=\left\langle P, \leq_{\mathcal{P}}\right\rangle$, we say that a set $A \subset P$ is cofinal in $\mathcal{P}$ if $\forall x \in P \exists y \in A\left(x \leq_{\mathcal{P}} y\right)$. The cofinality of $\mathcal{P}, \operatorname{cf}(\mathcal{P})$, is the least cardinal $\kappa$ such that there is a cofinal set of $\mathcal{P}$ of size $\kappa$. A regular cardinal is one whose cofinality is the cardinal itself. Note that if $\kappa$ is a regular cardinal and $\left\{x_{\gamma}: \gamma \in \kappa\right\}$ is an increasing sequence cofinal in $\mathcal{P}$, then $\mathcal{P}$ has cofinality $\kappa$.

Definition 1.7. Given an indecomposable linear ordering $\mathcal{L}$, let $\kappa$ be the cofinality of $\mathcal{L}^{\varepsilon_{\mathcal{L}}}$, and define $\tau(\mathcal{L})=\kappa^{\varepsilon_{\mathcal{L}}}$.

Note that if $\mathcal{L}$ is as above, it can be written as a sum $\mathcal{L}=\sum_{i \in \tau(\mathcal{L})} \mathcal{L}_{i}$ where the $\mathcal{L}_{i}$ have rank smaller than $\mathcal{L}$. Using Theorem 1.4(1), we can assume that $\mathcal{L}_{i} \in \mathbb{H}$ for each $i$.

Lemma 1.8. Let $\mathcal{L} \in \mathbb{H}$ and suppose that $\mathcal{L} \preccurlyeq \sum_{i \in \mathcal{A}} \mathcal{B}_{i}$.

(1) If $\tau(\mathcal{L}) \npreceq \mathcal{A}$, then $\mathcal{L} \preccurlyeq \mathcal{B}_{i}$ for some $i \in \mathcal{A}$.

(2) If $\varepsilon_{\mathcal{L}}=+$ and $\tau(\mathcal{L})<\operatorname{cf}(\mathcal{A})$, then there is an initial segment $\mathcal{A}_{0}$ of $\mathcal{A}$, of cofinality either $\tau(\mathcal{L})$ or 1 , such that $\mathcal{L} \preccurlyeq \sum_{i \in \mathcal{A}_{0}} \mathcal{B}_{i}$.

(3) If $\mathcal{A} \preccurlyeq \tau(\mathcal{L})$ and $1+\mathcal{L}+1 \preccurlyeq \sum_{i \in \mathcal{A}} \mathcal{B}_{i}$, then $\mathcal{L} \preccurlyeq \mathcal{B}_{i}$ for some $i \in \mathcal{A}$.

Proof. Suppose that $\varepsilon_{\mathcal{L}}=+$. Let $g$ be an embedding $\mathcal{L} \rightarrow \sum_{i \in \mathcal{A}} \mathcal{B}_{i}$, and let $\left\{x_{\gamma}: \gamma \in \tau(\mathcal{L})\right\}$ be an increasing cofinal sequence in $\mathcal{L}$. For each $\gamma<\tau(\mathcal{L})$, let $a_{\gamma} \in \mathcal{A}$ be such that $g\left(x_{\gamma}\right) \in \mathcal{B}_{a_{\gamma}}$. 
In the first case, there is a $\delta<\tau(\mathcal{L})$ such that $\forall \gamma \geq \delta\left(a_{\gamma}=a_{\delta}\right)$. Then, since $\mathcal{L}$ is indecomposable to the right, we have $\mathcal{L} \preccurlyeq \mathcal{B}_{a_{\delta}}$.

In the second case, let $\mathcal{A}_{0}=\left\{y \in \mathcal{A}: \exists \gamma<\tau(\mathcal{L})\left(y \leq_{\mathcal{A}} a_{\gamma}\right)\right\}$. Note that $\mathcal{L} \preccurlyeq \sum_{i \in \mathcal{A}_{0}} \mathcal{B}_{i}$. If there is an $a_{\delta}$ as above, then it is the maximal element of $\mathcal{A}_{0}$ and $\operatorname{cf}\left(\mathcal{A}_{0}\right)=1$. Otherwise, $\operatorname{cf}\left(\mathcal{A}_{0}\right)=\tau(\mathcal{L})$.

The last part follows from the first one.

Lemma 1.9. Let $\mathcal{L} \in \mathbb{H}$. Then $\tau(\mathcal{L})=(\operatorname{cf}(\operatorname{rk}(\mathcal{L})) \vee \omega)^{\varepsilon^{\mathcal{L}}}$. (Here, $\alpha \vee \beta$ denotes the maximum of $\alpha$ and $\beta$.)

Proof. Without loss of generality suppose that $\varepsilon_{\mathcal{L}}=+$ and assume that $\mathcal{L}$ has a first element $a$. If $\operatorname{rk}(\mathcal{L})$ is a successor ordinal, then $\mathcal{L}$ is an $\omega$-sum of smaller indecomposables, so $\tau(\mathcal{L})=\omega$. So, suppose that $\kappa=\operatorname{cf}(\operatorname{rk}(\mathcal{L})) \geq \omega$, and let $\left\{\alpha_{\gamma}: \gamma<\kappa\right\}$ be increasing and cofinal in $\operatorname{rk}(\mathcal{L})$. For each $\gamma<\kappa$, let $x_{\gamma} \in \mathcal{L}$ be such that $a \nsim_{\alpha_{\gamma}} x_{\gamma}$ and $\forall \delta<\gamma\left(x_{\delta} \leq_{\mathcal{L}} x_{\gamma}\right)$. We have constructed an increasing, cofinal sequence in $\mathcal{L}$ of size $\kappa$. Therefore $\tau(\mathcal{L})=\kappa$.

We let $\mathcal{R}_{e g^{ \pm}}$be the class of linear orderings $\tau$ such that either $\tau$ or $\tau^{*}$ is a regular cardinal.

Lemma 1.10. Suppose that $\mathcal{L} \in \mathbb{H}, \varepsilon_{\mathcal{L}}=+$, and $\left\{\mathcal{L}_{\gamma}: \gamma \in \alpha\right\}$ is such that $\mathcal{L}_{\gamma}+1 \preccurlyeq \mathcal{L}$ for each $\gamma$, and $\alpha \prec \tau(\mathcal{L})$. Then $\left(\sum_{\gamma \in \alpha} \mathcal{L}_{\gamma}\right)+1 \prec \mathcal{L}$. If $\alpha=\tau(\mathcal{L})$, then $\sum_{\gamma \in \alpha} \mathcal{L}_{\gamma} \preccurlyeq \mathcal{L}$.

Sketch of proof. Use transfinite induction on $\alpha$. When $\alpha$ is limit, use the fact that $\alpha<\operatorname{cf}(\mathcal{L})$.

Lemma 1.11. Let $\mathcal{A}, \mathcal{B}, \mathcal{C}$ and $\mathcal{D}$ be linear orderings such that $\mathcal{A} \cdot \mathcal{B} \preccurlyeq \mathcal{C} \cdot \mathcal{D}$ and $\mathcal{A}$ has either a first or a last element. Then either $\mathcal{A} \preccurlyeq \mathcal{C}$ or $\mathcal{B} \preccurlyeq \mathcal{D}$.

Proof. Let $a$ be either the first or the last element of $\mathcal{A}$, and let $g: \mathcal{A} \cdot \mathcal{B} \rightarrow$ $\mathcal{C} \cdot \mathcal{D}$ be an embedding. Suppose $\mathcal{B} \npreceq \mathcal{D}$. Then there has to exist $b_{0}<_{\mathcal{B}} b_{1} \in \mathcal{B}$ such that $g\left(\left\langle a, b_{0}\right\rangle\right)$ and $g\left(\left\langle a, b_{1}\right\rangle\right)$ belong to the same copy of $\mathcal{C}$. It follows that $\mathcal{A} \preccurlyeq \mathcal{C}$.

2. The invariants. In this section we show that the invariants $\operatorname{Inv}(\cdot)$ defined in the introduction behave well. First we show that an indecomposable linear ordering $\mathcal{L}$ is determined by $\varepsilon_{\mathcal{L}}$ and $\mathbb{I}_{\mathcal{L}}$. This result is essential for showing that different equimorphism types get different invariants. Then we show how to define an ordering on $\mathcal{T} r$ which coincides with the embeddability relation on indecomposables. We use this relation to define an ordering on $\mathcal{I} n$ which coincides with the embeddability relation on scattered linear orderings.

In the last subsection we characterize the finite trees with labels in $\mathcal{O} n \times$ $\{-,+\}$ which belong to $\mathcal{T}_{r}$, and the sequences in $\mathcal{T}_{r}<\omega$ which belong to $\mathcal{I}_{n}$. 
2.1. Equimorphism invariants. We start by defining the Hausdorff rank of an ideal and proving some of its basic properties.

Definition 2.1. Given an ideal $\mathbb{I} \subset \mathbb{H}$, we define its $\operatorname{rank}$ to be $\operatorname{rk}(\mathbb{I})=$ $\sup \{\operatorname{rk}(\mathcal{L})+1: \mathcal{L} \in \mathbb{I}\}$.

Lemma 2.2. Let $\mathcal{L} \in \mathbb{H}$. Then

(1) $\operatorname{rk}\left(\mathbb{I}_{\mathcal{L}}\right)=\operatorname{rk}(\mathcal{L})$

(2) if $\operatorname{cf}\left(\mathbb{I}_{\mathcal{L}}\right)>\omega$, then $\operatorname{cf}\left(\mathbb{I}_{\mathcal{L}}\right)=\operatorname{cf}\left(\operatorname{rk}\left(\mathbb{I}_{\mathcal{L}}\right)\right)$;

(3) if $\operatorname{cf}\left(\mathbb{I}_{\mathcal{L}}\right) \leq \omega$, then $\operatorname{cf}\left(\operatorname{rk}\left(\mathbb{I}_{\mathcal{L}}\right)\right) \leq \omega$.

Proof. Write $\mathcal{L}$ as $\sum_{\gamma \in \tau(\mathcal{L})} \mathcal{L}_{\gamma}$ where each $\mathcal{L}_{\gamma} \in \mathbb{H}$. Without loss of generality assume that $\varepsilon_{\mathcal{L}}=+$ and let $\kappa=\tau(\mathcal{L})=\operatorname{cf}(\operatorname{rk}(\mathcal{L})) \vee \omega$.

Suppose first that $\kappa>\omega$. For each $\gamma \in \kappa$, we define $\alpha_{\gamma}<\kappa$ and $\mathcal{L}^{\gamma} \in \mathbb{H}$ by transfinite recursion as follows. Let $\alpha_{0}=1$ and $\mathcal{L}^{0}=\mathcal{L}_{0}$. Let $\alpha_{\gamma}$ be such that for every $\delta<\gamma, \alpha_{\delta}<\alpha_{\gamma}$ and $\mathcal{L}^{\delta} \prec \sum_{\beta<\alpha_{\gamma}} \mathcal{L}_{\beta}$. Let $\mathcal{L}^{\gamma}=\left(\sum_{\delta<\alpha_{\gamma}} \mathcal{L}_{\delta}\right) \cdot \omega$. Note that, since $\mathcal{L}$ is indecomposable to the right, $\mathcal{L}^{\gamma} \preccurlyeq \mathcal{L}$. The existence of $\alpha_{\gamma}$ follows from Lemma 1.8. It also follows that $\mathcal{L}^{\gamma} \in \mathbb{I}_{\mathcal{L}}$. Also, if $\mathcal{A} \in \mathbb{I}_{\mathcal{L}}$, then $\mathcal{A} \preccurlyeq \mathcal{L}^{\gamma}$ for some $\gamma \in \kappa$. So $\left\{\mathcal{L}^{\gamma}: \gamma \in \kappa\right\}$ is an increasing cofinal sequence in $\mathbb{I}_{\mathcal{L}}$ of size $\kappa$, and hence $\operatorname{cf}\left(\mathbb{I}_{\mathcal{L}}\right)=\kappa$ and $\operatorname{rk}\left(\mathbb{I}_{\mathcal{L}}\right)=\sup \left\{\operatorname{rk}\left(\mathcal{L}^{\gamma}\right)+1: \gamma \in \kappa\right\} \leq$ $\operatorname{rk}(\mathcal{L})$. For any two $x, y \in \mathcal{L}$, the interval $[x, y]_{\mathcal{L}}$ embeds into $\mathcal{L}^{\gamma}$ for some $\gamma \in \kappa$, and hence $x \approx_{\operatorname{rk}\left(\mathcal{L}^{\gamma}\right)+1} y$. Therefore $\operatorname{rk}(\mathcal{L}) \leq \sup \left\{\operatorname{rk}\left(\mathcal{L}^{\gamma}\right)+1: \gamma \in \kappa\right\}$, and hence $\operatorname{rk}\left(\mathbb{I}_{\mathcal{L}}\right)=\operatorname{rk}(\mathcal{L})$.

Suppose now that $\kappa=\omega$. Then it follows from Lemma 1.8(3) that $\left\{\mathcal{L}_{\gamma}: \gamma \in \omega\right\}$ is cofinal in $\mathbb{I}_{\mathcal{L}}$ and hence $\operatorname{cf}\left(\mathbb{I}_{\mathcal{L}}\right) \leq \omega$ and $\operatorname{rk}\left(\mathbb{I}_{\mathcal{L}}\right)=$ $\sup \left\{\operatorname{rk}\left(\mathcal{L}_{\gamma}\right)+1: \gamma \in \omega\right\} \leq \operatorname{rk}(\mathcal{L})$. For any two $x, y \in \mathcal{L}$, the interval $[x, y]_{\mathcal{L}}$ embeds into a finite sum of $\mathcal{L}^{\gamma}$ 's, and hence $x \approx_{\sup \left\{\operatorname{rk}\left(\mathcal{L}_{\gamma}\right)+1: \gamma \in \omega\right\}} y$. It follows that $\operatorname{rk}(\mathcal{L}) \leq \sup \left\{\operatorname{rk}\left(\mathcal{L}^{\gamma}\right)+1: \gamma \in \kappa\right\}$, and hence $\operatorname{rk}\left(\mathbb{I}_{\mathcal{L}}\right)=\operatorname{rk}(\mathcal{L})$.

Definition 2.3. Given an ideal $\mathbb{I} \subset \mathbb{H}$ and a $\tau \in \mathcal{R} e g^{ \pm}$, we say that a linear ordering $\mathcal{L}$ is a $\tau$-unbounded sum of $\mathbb{I}$, and we write $\mathcal{L}=\mathbb{I} \cdot \tau$, if $\mathcal{L}$ can be written as

$$
\mathcal{L}=\sum_{i \in \tau} \mathcal{B}_{i}
$$

where $\left\{\mathcal{B}_{i}: i \in \tau\right\} \subseteq \mathbb{I}$, and any other linear ordering of that form embeds in $\mathcal{L}$. Note that, up to equimorphism, there exists at most one $\tau$-unbounded sum of $\mathbb{I}$.

The idea for the definition above came from Laver's regular unbounded sums and shuffle sums [Lav71].

The $\tau$-unbounded sum of an ideal $\mathbb{I}$ may not exist. But in some cases we know it does exist:

Lemma 2.4. Let $\mathbb{I} \subset \mathbb{H}$ be an ideal and $\tau \in \mathcal{R} e g^{ \pm}$be such that $\operatorname{cf}(\mathbb{I}) \leq|\tau|$. Then the unbounded sum $\mathbb{I} \cdot \tau$ exists. 
Proof. Let $\kappa=|\tau|$ and let $\left\{\mathcal{L}_{\xi}: \xi<\kappa\right\}$ be cofinal in $\mathbb{I}$. Let $\pi: \tau \rightarrow \kappa$ be a function such that for every $\delta \in \kappa$, the set $\pi^{-1}[\delta]$ has size $\kappa$. Let $\mathcal{L}$ be the equimorphism type of $\sum_{\gamma \in \tau} \mathcal{L}_{\pi(\gamma)}$. Using Lemma 1.10, it is not hard to prove that if $\mathcal{B}=\sum_{i \in \tau} \mathcal{B}_{i}$, and $\left\{\mathcal{B}_{i}: i \in \tau\right\} \subseteq \mathbb{I}$, then $\mathcal{B} \preccurlyeq \mathcal{L}$. So, $\mathcal{L}$ is the $\tau$-unbounded sum of $\mathbb{I}$.

Corollary 2.5. Let $\mathcal{L} \in \mathbb{H}$. Then $\mathbb{I}_{\mathcal{L}} \cdot \tau(\mathcal{L})$ exists and equals $\mathcal{L}$.

Proof. Clearly $\mathcal{L} \preccurlyeq \mathbb{I}_{\mathcal{L}} \cdot \tau(\mathcal{L})$. That $\mathbb{I}_{\mathcal{L}} \cdot \tau(\mathcal{L}) \preccurlyeq \mathcal{L}$ follows from Lemma 1.10.

Corollary 2.6. If $\mathcal{A}, \mathcal{B} \in \mathbb{H}$ are such that $\varepsilon_{\mathcal{A}}=\varepsilon_{\mathcal{B}}$ and $\mathbb{I}_{\mathcal{A}}=\mathbb{I}_{\mathcal{B}}$, then $\mathcal{A}=\mathcal{B}$.

Proof. We know that $\tau(\mathcal{A})$ depends only on $\operatorname{rk}(\mathcal{A})$ and $\varepsilon_{\mathcal{A}}$ and that $\operatorname{rk}(\mathcal{A})=\operatorname{rk}\left(\mathbb{I}_{\mathcal{A}}\right)$. So, $\tau(\mathcal{A})=\tau(\mathcal{B})$. But then $\mathcal{A}=\mathbb{I}_{\mathcal{A}} \cdot \tau(\mathcal{A})=\mathbb{I}_{\mathcal{B}} \cdot \tau(\mathcal{B})=\mathcal{B}$.

It follows that the invariants $\mathrm{T}(\cdot)$ defined in the introduction are one-toone on $\mathbb{H}$. Thus, the invariants $\operatorname{Inv}(\cdot)$ are one-to-one on $\mathbb{S}$.

2.2. Ordering of invariants. In this subsection we define a relation $\preccurlyeq$ on $\mathcal{T} r$ such that $\mathrm{T}$ is an isomorphism between $\langle\mathbb{H}, \preccurlyeq\rangle$ and $\left\langle\mathcal{T}_{r}, \preccurlyeq\right\rangle$. We then define a relation $\preccurlyeq$ on $\mathcal{I}_{n}$ such that Inv : $\langle\mathbb{S}, \preccurlyeq\rangle \rightarrow\left\langle\mathcal{I}_{n}, \preccurlyeq\right\rangle$ is an isomorphism.

Notation 2.7. Given a tree $T=\left[\left\langle\beta, \varepsilon_{T}\right\rangle ; T_{0}, \ldots, T_{k-1}\right]$, let $\operatorname{rk}(T)=\beta$ and $\tau(T)=\operatorname{cf}(\beta)^{\varepsilon_{T}}$.

Definition 2.8. Given $S=\left[\left\langle\alpha, \varepsilon_{S}\right\rangle ; S_{0}, \ldots, S_{l-1}\right]$ and $T=\left[\left\langle\beta, \varepsilon_{T}\right\rangle\right.$; $\left.T_{0}, \ldots, T_{k-1}\right] \in \mathcal{T} r$ we let $S \preccurlyeq T$ if either

- $\alpha \leq \beta, \tau(S) \preccurlyeq \tau(T)$ and $\forall i<k\left(\operatorname{rk}\left(T_{i}\right) \geq \alpha \vee \exists j<l\left(S_{j} \preccurlyeq T_{i}\right)\right)$, or

- $\alpha<\beta, \tau(S) \npreceq \tau(T)$ and $\forall i<k\left(T_{i} \npreceq S\right)$.

Proposition 2.9. For $\mathcal{A}, \mathcal{B} \in \mathbb{H}, \mathcal{A} \preccurlyeq \mathcal{B}$ if and only if $\mathrm{T}(\mathcal{A}) \preccurlyeq \mathrm{T}(\mathcal{B})$.

Proof. The key point is that $\mathcal{A} \preccurlyeq \mathcal{B}$ if and only if either

- $\tau(\mathcal{A}) \preccurlyeq \tau(\mathcal{B})$ and $\mathbb{I}_{\mathcal{A}} \subseteq \mathbb{I}_{\mathcal{B}}$, or

- $\tau(\mathcal{A}) \nprec \tau(\mathcal{B})$ and $\mathcal{A} \in \mathbb{I}_{\mathcal{B}}$.

The reason is the following. If $\mathcal{A} \preccurlyeq \mathcal{B}$, then clearly $\mathbb{I}_{\mathcal{A}} \subseteq \mathbb{I}_{\mathcal{B}}$, and if $\tau(\mathcal{A}) \npreceq$ $\tau(\mathcal{B})$ then $\mathcal{A} \in \mathbb{I}_{\mathcal{B}}$ by Lemma 1.8(1). Conversely, if $\mathcal{A} \in \mathbb{I}_{\mathcal{B}}$ then clearly $\mathcal{A} \preccurlyeq \mathcal{B}$, and if $\tau(\mathcal{A}) \preccurlyeq \tau(\mathcal{B})$ and $\mathbb{I}_{\mathcal{A}} \subseteq \mathbb{I}_{\mathcal{B}}$ then $\mathcal{A} \preccurlyeq \mathcal{B}$ by Lemma 1.10.

The proposition then follows from the following observation. Let $\alpha=$ $\operatorname{rk}(\mathcal{A})$ and $\beta=\operatorname{rk}(\mathcal{B})$, let $\left\{\mathcal{A}_{0}, \ldots, \mathcal{A}_{l-1}\right\}$ be the set of minimal elements of $\mathbb{H}_{\alpha} \backslash \mathbb{I}_{\mathcal{A}}$, and let $\left\{\mathcal{B}_{0}, \ldots, \mathcal{B}_{k-1}\right\}$ be the set of minimal elements of $\mathbb{H}_{\beta} \backslash \mathbb{I}_{\mathcal{B}}$. Then $\mathbb{I}_{\mathcal{A}} \subseteq \mathbb{I}_{\mathcal{B}}$ if and only if $\alpha \leq \beta$ and for each $i<k$, either $\mathcal{B}_{i} \notin \mathbb{H}_{\alpha}$ or there exists $j<l$ such that $\mathcal{A}_{j} \preccurlyeq \mathcal{B}_{i}$. Also, $\mathcal{A} \in \mathbb{I}_{\mathcal{B}}$ if and only if $\alpha<\beta$ and $\mathcal{B}_{i} \npreceq \mathcal{A}$ for each $i<k$. 
Definition 2.10. Given $S=\left\langle S_{0}, \ldots, S_{l}\right\rangle \in \mathcal{I} n$ and $T=\left[\left\langle\alpha, \varepsilon_{T}\right\rangle ;\right.$ $\left.T_{0}, \ldots, T_{k-1}\right] \in \mathcal{T} r$ we let $S \preccurlyeq T$ if either

- $\varepsilon_{T}=+, S_{l} \preccurlyeq T$ and $\forall j \in\{0, \ldots, l-1\}\left(\operatorname{rk}\left(S_{j}\right)<\alpha \& \forall i<k\right.$ $\left.\left(T_{i} \npreceq S_{j}\right)\right)$, or

- $\varepsilon_{T}=-, S_{0} \preccurlyeq T$ and $\forall j \in\{1, \ldots, l\}\left(\operatorname{rk}\left(S_{j}\right)<\alpha \& \forall i<k\left(T_{i} \npreceq S_{j}\right)\right)$.

Lemma 2.11. Let $\mathcal{A} \in \mathbb{S}$ and $\mathcal{L} \in \mathbb{H}$. Then $\operatorname{Inv}(\mathcal{A}) \preccurlyeq \mathrm{T}(\mathcal{L})$ if and only if $\mathcal{A} \preccurlyeq \mathcal{L}$.

Proof. Write $\mathcal{A}$ as a sum of indecomposables, $\mathcal{A}_{0}+\cdots+\mathcal{A}_{l}$, and assume without loss of generality that $\varepsilon_{\mathcal{L}}=+$. Note that $\operatorname{Inv}(\mathcal{A}) \preccurlyeq \mathrm{T}(\mathcal{L})$ if and only if $\mathcal{A}_{l} \preccurlyeq \mathcal{L}$ and $\mathcal{A}_{j}+1 \preccurlyeq \mathcal{L}$ for every $j=0, \ldots, l-1$. So, it is clear that if $\mathcal{A} \preccurlyeq \mathcal{L}$, then $\operatorname{Inv}(\mathcal{A}) \preccurlyeq \mathrm{T}(\mathcal{L})$. Suppose now that $\operatorname{Inv}(\mathcal{A}) \preccurlyeq \mathrm{T}(\mathcal{L})$. Then, by Lemma $1.10, \sum_{j=0}^{l-1} \mathcal{A}_{j}+1 \preccurlyeq \mathcal{L}$. Since $\mathcal{A}_{l} \preccurlyeq \mathcal{L}$ and $\mathcal{L}$ is indecomposable to the right, we conclude that $\mathcal{A} \preccurlyeq \mathcal{L}$.

Definition 2.12. Now, given $S=\left\langle S_{0}, \ldots, S_{l}\right\rangle \in \mathcal{I} n$ and $T=\left\langle T_{0}, \ldots, T_{k}\right\rangle$ $\in \mathcal{I} n$ we let $S \preccurlyeq T$ if

$$
\bigvee_{0=i_{0} \leq \cdots \leq i_{k} \leq i_{k+1}=l+1}\left(\bigwedge_{n \leq k}\left\langle S_{i_{n}}, S_{i_{n}+1}, \ldots, S_{i_{n+1}-1}\right\rangle \preccurlyeq T_{n}\right) .
$$

Proposition 2.13. Let $\mathcal{A}, \mathcal{B} \in \mathbb{S}$. Then $\operatorname{Inv}(\mathcal{A}) \preccurlyeq \operatorname{Inv}(\mathcal{B})$ if and only if $\mathcal{A} \preccurlyeq \mathcal{B}$.

Proof. Let $\mathcal{A}=\mathcal{A}_{0}+\cdots+\mathcal{A}_{l}$ and $\mathcal{B}=\mathcal{B}_{0}+\cdots+\mathcal{B}_{k}$, and let $S=$ $\left\langle S_{0}, \ldots, S_{l}\right\rangle=\operatorname{Inv}(\mathcal{A})$ and $T=\left\langle T_{0}, \ldots, T_{k}\right\rangle=\operatorname{Inv}(\mathcal{B})$, where $S_{i}=\mathrm{T}\left(\mathcal{A}_{i}\right)$ and $T_{n}=\mathrm{T}\left(\mathcal{B}_{n}\right)$. Note that $\operatorname{Inv}(\mathcal{A}) \preccurlyeq \operatorname{Inv}(\mathcal{B})$ if and only if for some tuple $i_{0}, \ldots, i_{k+1}$ with $0=i_{0} \leq \cdots \leq i_{k} \leq i_{k+1}=l+1$ we have

$$
\begin{array}{r}
\mathcal{A}_{0}+\cdots+\mathcal{A}_{i_{1}-1} \preccurlyeq \mathcal{B}_{0} \& \cdots \& \mathcal{A}_{i_{n}}+\cdots+\mathcal{A}_{i_{n}-1} \preccurlyeq \mathcal{B}_{n} \\
\& \cdots \& \mathcal{A}_{i_{k}}+\cdots+\mathcal{A}_{l} \preccurlyeq \mathcal{B}_{k} .
\end{array}
$$

It is then clear that if $\operatorname{Inv}(\mathcal{A}) \preccurlyeq \operatorname{Inv}(\mathcal{B})$, then $\mathcal{A} \preccurlyeq \mathcal{B}$.

Suppose now that $\mathcal{A} \preccurlyeq \mathcal{B}$ and let $f: \mathcal{A} \rightarrow \mathcal{B}$ be an embedding. For each $i$, let $f_{i}$ be the restriction of $\mathcal{A}$ to $\mathcal{A}_{i}$. Since each $\mathcal{A}_{i}$ is indecomposable, there exists an embedding $g_{i}: \mathcal{A}_{i} \rightarrow \mathcal{B}$ such that the image of $g_{i}$ is included in the image of $f_{i}$ and also in $\mathcal{B}_{n}$ for some $n$. Putting these $g_{i}$ 's together we get an embedding $g: \mathcal{A} \rightarrow \mathcal{B}$ such that the image of each $\mathcal{A}_{i}$ is included in some $\mathcal{B}_{n}$. For each $n$, let $i_{n}$ be the first $i$ such that the image of $\mathcal{A}_{i}$ under $g$ is included in $B_{n}$. Then (1) follows, and hence $\operatorname{Inv}(\mathcal{A}) \preccurlyeq \operatorname{Inv}(\mathcal{B})$.

2.3. The class of invariants. Now we are interested in characterizing $\mathcal{T}_{r}$ and $\mathcal{I}_{n}$. Given an ordinal $\alpha$ and $T_{0}, \ldots, T_{k-1} \in \mathcal{I} r$, let

$$
\mathcal{I}_{T_{0}, \ldots, T_{k-1}}^{\alpha}=\left\{S \in \mathcal{T} r: \operatorname{rk}(S)<\alpha \& \forall i<k\left(T_{i} \npreceq S\right)\right\} .
$$

Given an ideal $\mathcal{I} \subset \mathcal{T} r$, let $\operatorname{rk}(\mathcal{I})=\sup \{\operatorname{rk}(T)+1: T \in \mathcal{I}\}$. 
Proposition 2.14. A tree $T=\left[\langle\alpha, \varepsilon\rangle ; T_{0}, \ldots, T_{k-1}\right]$ with labels in $\mathcal{R}_{e} g^{ \pm}$ belongs to $\mathcal{T r}$ if and only if

(1) for each $i, T_{i} \in \mathcal{T}$ r and $\operatorname{rk}\left(T_{i}\right)<\alpha$;

(2) $T_{0}, \ldots, T_{k-1}$ are mutually $\preccurlyeq$-incomparable;

(3) $\operatorname{rk}\left(\mathcal{I}_{T_{0}, \ldots, T_{k-1}}^{\alpha}\right)=\alpha$;

(4) $\operatorname{cf}\left(\mathcal{I}_{T_{0}, \ldots, T_{k-1}}^{\alpha}\right) \vee \omega=\operatorname{cf}(\alpha) \vee \omega$;

(5) for no $i, \tau\left(T_{i}\right) \prec \tau(T)$.

Proof. First we prove the "only if" part. Suppose $T=\mathrm{T}(\mathcal{L})$ with $\mathcal{L} \in \mathbb{H}$. The first two assertions are obvious from the definition of $\mathrm{T}$. The third and fourth follow from Lemma 2.2. For the last item suppose, toward a contradiction, that $\varepsilon=+, \tau(T)=\kappa$ and $\tau\left(T_{i}\right)=\lambda<\kappa$. Let $\mathcal{L}_{i}$ be the minimal element of $\mathbb{H}_{\alpha} \backslash \mathbb{I}_{\mathcal{L}}$ such that $T_{i}=\mathrm{T}\left(\mathcal{L}_{i}\right)$. Then $\mathcal{L}_{i}=\sum_{j \in \lambda} \mathcal{L}_{i, j}$ for some $\mathcal{L}_{i, j} \in \mathbb{I}_{\mathcal{L}_{i}}$. For every $j, \mathcal{L}_{i, j} \prec \mathcal{L}_{i}$ and hence $\mathcal{L}_{i, j}$ belongs to $\mathbb{I}_{\mathcal{L}}$. By Lemma 1.10, $\left(\sum_{\lambda} \mathcal{L}_{i, j}\right)+1 \preccurlyeq \mathcal{L}$. Therefore $\mathcal{L}_{i} \in \mathbb{I}_{\mathcal{L}}$, contradicting its choice.

Let us now prove the other direction. Suppose that $T$ satisfies the five conditions above. Let $\mathbb{I}=\left\{\mathcal{A} \in \mathbb{H}_{\alpha}: \mathrm{T}(\mathcal{A}) \in \mathcal{I}_{T_{0}, \ldots, T_{k-1}}^{\alpha}\right\}$. This ideal has rank $\alpha$ and cofinality $\operatorname{cf}(\alpha)$. Let $\tau=\operatorname{cf}(\alpha)^{\varepsilon}=\tau(T)$. Let $\mathcal{L}=\mathbb{I} \cdot \tau$. We claim that $T=\mathrm{T}(\mathcal{L})$. Clearly $\varepsilon_{\mathcal{L}}=\varepsilon, \operatorname{rk}(\mathcal{L})=\alpha$ and $\mathbb{I} \subseteq \mathbb{I}_{\mathcal{L}}$. Suppose toward a contradiction that $\mathbb{I}_{\mathcal{L}} \nsubseteq \mathbb{I}$. Let $\mathcal{L}_{i}$ be a minimal element of $\mathbb{I}_{\mathcal{L}} \backslash \mathbb{I}$. In particular, $\mathcal{L}_{i}$ is a minimal element of $\mathbb{H}_{\alpha} \backslash \mathbb{I}$, so $\mathrm{T}\left(\mathcal{L}_{i}\right)=T_{i}$ for some $i<k$. Since $\tau\left(\mathcal{L}_{i}\right)=\tau\left(T_{i}\right) \nprec \tau$ and $1+\mathcal{L}_{i}+1 \prec \mathcal{L}$, we find that $\mathcal{L}_{i}$ embeds into one of the summands of $\mathbb{I} \cdot \tau(\mathcal{L})$, and hence belongs to $\mathbb{I}$. This contradicts the choice of $\mathcal{L}_{i}$.

Notation 2.15. If $T=\left[\langle\alpha, \varepsilon\rangle ; T_{0}, \ldots, T_{k-1}\right] \in \mathcal{T} r$, let $\mathcal{I}_{T}=\mathcal{I}_{T_{0}, \ldots, T_{k-1}}^{\alpha}$.

Proposition 2.16. Let $T=\left\langle T_{0}, \ldots, T_{k}\right\rangle \in \mathcal{T}_{r}<\omega$. Then $T \in \mathcal{I}$ if and only if for any $i<k$ we have neither

(1) $\varepsilon_{i}=-$ and $T_{i+1} \in \mathcal{I}_{T_{i}}$, nor

(2) $\varepsilon_{i+1}=+$ and $T_{i} \in \mathcal{I}_{T_{i+1}}$.

Sketch of proof. Let $\mathcal{A}_{0}, \ldots, \mathcal{A}_{k} \in \mathbb{H}$ be such that $\mathrm{T}\left(\mathcal{A}_{i}\right)=T_{i}$. Then $\left\langle T_{0}, \ldots, T_{k}\right\rangle \in \operatorname{Inv}$ if and only if $\left\langle\mathcal{A}_{0}, \ldots, \mathcal{A}_{k}\right\rangle$ is a minimal decomposition of $\mathcal{A}_{0}+\cdots+\mathcal{A}_{k}$. It is not hard to see that if $\left\langle\mathcal{A}_{0}, \ldots, \mathcal{A}_{k}\right\rangle$ is not a minimal decomposition, then for some $i$, either $\mathcal{A}_{i}+\mathcal{A}_{i+1} \preccurlyeq \mathcal{A}_{i}$ or $\mathcal{A}_{i}+\mathcal{A}_{i+1} \preccurlyeq \mathcal{A}_{i+1}$. Now, $\mathcal{A}_{i}+\mathcal{A}_{i+1} \preccurlyeq \mathcal{A}_{i+1}$ if and only if $\varepsilon_{\mathcal{A}_{i+1}}=+$ and $\mathcal{A}_{i}+1 \preccurlyeq \mathcal{A}_{i+1}$, while $\mathcal{A}_{i}+\mathcal{A}_{i+1} \preccurlyeq \mathcal{A}_{i}$ if and only if $\varepsilon_{\mathcal{A}_{i+1}}=-$ and $1+\mathcal{A}_{i+1} \preccurlyeq \mathcal{A}_{i}$. The proposition follows.

We note that all the conditions in the two propositions above can be easily checked via a finite manipulation of symbols, using maybe some basic operations on ordinals, except for 2.14(3) and 2.14(4). We will prove in the next sections that condition 2.14(3) can also be checked using such an 
algorithm (see the end of Section 4). But we know nothing about condition 2.14(4). For the countable case, it always holds, and hence we can tell whether a sequence belongs to $\mathcal{I} n_{\omega_{1}}$ or not, using a finite algorithm. As a corollary we find that if $\alpha$ is a computable ordinal, then $\mathbb{S}_{\alpha}$ is computably presentable [Mon05b, Corollary 4.3]. We could use this result to prove that every equimorphism type in $\mathbb{S}_{\alpha}$ has a computably presentable member, getting a different proof of [Mon05b, Theorem 2.3].

3. Minimal linear orderings. In this section we explicitly define the elements of $\mathbb{F}_{\alpha}$ for each $\alpha$, where $\mathbb{F}_{\alpha}$ is the set of minimal equimorphism types of rank $\alpha$. Also, for each $\alpha$, we explicitly define a finite set of ideals of $\mathbb{H}$ which contains the set of minimal ideals of rank $\alpha$.

Definition 3.1. Given an indecomposable ordinal $\alpha>1$, and two signs $\varepsilon_{0}$ and $\varepsilon_{1}$, we define an equimorphism type $\operatorname{lin}\left(\left\langle\alpha, \varepsilon_{0}, \varepsilon_{1}\right\rangle\right)$ as follows. Let $\left\{\alpha_{\gamma}: \gamma<\operatorname{cf}(\alpha)\right\}$ be an increasing sequence cofinal in $\alpha$. Define

$$
\operatorname{lin}\left(\left\langle\alpha, \varepsilon_{0}, \varepsilon_{1}\right\rangle\right)=\sum_{\gamma \in \operatorname{cf}(\alpha)^{\varepsilon_{1}}}\left(\omega^{\alpha_{\gamma}}\right)^{\varepsilon_{0}} .
$$

Observe that, up to equimorphism, this definition is independent of the cofinal sequence chosen. We also let $\operatorname{lin}(\langle 1,+\rangle)=\omega$ and $\operatorname{lin}(\langle 1,-\rangle)=\omega^{*}$. We call these equimorphism types basic linear orderings. We use b.1.o. to denote the set of codes for basic linear orderings:

$$
\begin{aligned}
\text { b.1.o. }= & \left\{\left\langle\alpha, \varepsilon_{0}, \varepsilon_{1}\right\rangle: \alpha=\omega^{\delta}, \delta \in \mathcal{O} n, \delta>0 \& \varepsilon_{0}, \varepsilon_{1} \in\{+,-\}\right\} \\
& \cup\{\langle 1,+\rangle,\langle 1,-\rangle\} .
\end{aligned}
$$

Finite products of basic linear orderings will be called finitely alternating linear orderings. Let F.1.o. = b.1.o. ${ }^{<\omega}$. Given $\vec{a}=\left\langle a_{0}, \ldots, a_{n}\right\rangle \in$ F.1.o., let

$$
\operatorname{lin}(\vec{a})=\operatorname{lin}\left(a_{0}\right) \cdots \operatorname{lin}\left(a_{n}\right) .
$$

ExAmple 3.2. $\operatorname{lin}(\langle\alpha,+,+\rangle)=\omega^{\alpha}$ and $\operatorname{lin}(\langle\alpha,-,-\rangle)=\omega^{\alpha *}$.

Notation 3.3. For $a=\left\langle\alpha, \varepsilon_{0}, \varepsilon_{1}\right\rangle \in$ b.1.o., let $\operatorname{rk}(a)=\alpha$ and $\varepsilon_{a}=\varepsilon_{1}$, and for $a=\langle 1, \varepsilon\rangle$, let $\operatorname{rk}(a)=1$ and $\varepsilon_{a}=\varepsilon$. Let $\operatorname{cf}(a)=\operatorname{cf}(\operatorname{rk}(a))$ and $\tau(a)=\operatorname{cf}(a)^{\varepsilon_{a}}$. Given $\vec{a}=\left\langle a_{0}, \ldots, a_{n}\right\rangle \in$ F.1.o., let $\operatorname{rk}(\vec{a})=\sum_{i \leq n} \operatorname{rk}\left(a_{i}\right)$, $\operatorname{cf}(\vec{a})=\operatorname{cf}\left(a_{n}\right), \varepsilon_{\vec{a}}=\varepsilon_{a_{n}}$, and $\tau(\vec{a})=\tau\left(a_{n}\right)$.

DeFinition 3.4. Let $\delta$ be an ordinal with Cantor normal form $\delta=\omega^{\alpha_{0}}+$ $\cdots+\omega^{\alpha_{k-1}}$ where $\alpha_{0} \geq \alpha_{1} \geq \cdots \geq \alpha_{k-1}$. Let

$$
\text { F.1.o. } \delta=\left\{\left\langle a_{0}, \ldots, a_{k-1}\right\rangle \in \text { F.1.o. }: \forall i<k\left(\operatorname{rk}\left(a_{i}\right)=\omega^{\alpha_{i}}\right)\right\} .
$$

Proposition 3.5. Let $\mathcal{L} \in \mathbb{H}$ and $\delta$ be an ordinal. Then

$$
\delta \leq \operatorname{rk}(\mathcal{L}) \Leftrightarrow(\exists \vec{a} \in \text { F.1.o. } \delta) \operatorname{lin}(\vec{a}) \preccurlyeq \mathcal{L} .
$$

To prove this proposition we need a few lemmas. 
Lemma 3.6. Let $\mathcal{L}$ be a scattered linear ordering of rank $\alpha$. If we color $\mathcal{L}$ with finitely many colors, then there is a monochromatic subset of $\mathcal{L}$ of rank $\alpha$. In other words, if $C$ is a finite set, then for every $f: \mathcal{L} \rightarrow C$, there exists $c \in C$ such that $\operatorname{rk}\left(f^{-1}[c]\right)=\alpha$.

Proof. We use transfinite induction on $\alpha$.

Suppose first that $\alpha$ is a limit ordinal. For every $\beta<\alpha$ there exists a color $c_{\beta}$ such that $\operatorname{rk}\left(f^{-1}\left[c_{\beta}\right]\right) \geq \beta$. There is some color $c$ such that $\{\beta<\alpha$ : $\left.c_{\beta}=c\right\}$ is unbounded in $\alpha$. Then $f^{-1}[c]$ has rank $\alpha$.

Suppose now that $\alpha=\beta+1$. Then $\mathcal{L}^{(\beta)}$ is infinite. Note that the set of $\approx_{\beta}$-equivalence classes which have rank $\beta$ is also infinite. For each $x \in \mathcal{L}^{(\beta)}$ that has rank $\beta$, there exists a color $c_{x}$ such that $f^{-1}\left[c_{x}\right] \cap x$ has rank $\beta$. (We are thinking of $x$ as a segment of $\mathcal{L}$.) For some color $c$, the set $\left\{x \in \mathcal{L}^{(\beta)}\right.$ : $\left.\operatorname{rk}(x)=\beta \& c_{x}=c\right\}$ is infinite. For that $c$ the set $f^{-1}[c]$ has rank $\alpha$.

Lemma 3.7. If $\mathcal{L}$ is a linear ordering of rank $\alpha$ and $\alpha=\beta+\gamma$, then there exist indecomposable linear orderings $\mathcal{B}$ and $\mathcal{C}$ of ranks $\beta$ and $\gamma$ respectively such that $\mathcal{B} \cdot \mathcal{C} \preccurlyeq \mathcal{L}$.

Proof. Consider $\mathcal{L}^{(\beta)}=\mathcal{L} / \approx_{\beta}$. Note that $\mathcal{L}=\sum_{x \in \mathcal{L}^{(\beta)}} x$, viewing each equivalence class as a segment of $\mathcal{L}$. Let

$$
\widehat{\mathcal{L}}=\sum_{x \in \mathcal{L}^{(\beta)}, \operatorname{rk}(x)=\beta} x \subseteq \mathcal{L} .
$$

Note that $\widehat{\mathcal{L}}^{(\beta)}=\left\{x \in \mathcal{L}^{(\beta)}: \operatorname{rk}(x)=\beta\right\}$.

We claim that $\operatorname{rk}(\widehat{\mathcal{L}})=\alpha$. For each $\delta \geq \beta$, let $f_{\delta}: \widehat{\mathcal{L}}^{(\delta)} \rightarrow \mathcal{L}^{(\delta)}$ be the obvious embedding: $f_{\delta}(y)$ is the unique $z \in \mathcal{L}^{(\delta)}$ such that $y \subseteq z$. We will prove that $f_{\delta}\left[\widehat{\mathcal{L}}^{(\delta)}\right]=\left\{z \in \mathcal{L}^{(\delta)}: \operatorname{rk}(z) \geq \beta\right\}$ for each $\delta \geq \beta$. Clearly $f_{\delta}\left[\widehat{\mathcal{L}}^{(\delta)}\right] \subseteq\left\{z \in \mathcal{L}^{(\delta)}: \operatorname{rk}(z) \geq \beta\right\}$. Now, suppose that $z \in \mathcal{L}^{(\delta)}$ and $\operatorname{rk}(z) \geq \beta$. Then $z$ can be written as a sum of $\approx_{\beta}$-equivalence classes in $\mathcal{L}^{(\beta)}$. If it is only one $\approx_{\beta}$ equivalence class, then the class belongs to $\widehat{\mathcal{L}}^{(\beta)}$, and $z=f_{\delta}(z) \in$

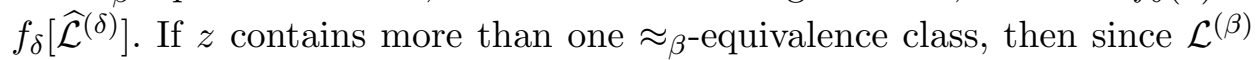
is scattered, there are $x, w \in \mathcal{L}^{(\beta)}$ with $x, w \subseteq z$ which are adjacent. Then at least one of $x$ and $w$ belongs to $\widehat{\mathcal{L}}^{(\beta)}$. This is because if $\operatorname{rk}(x)<\beta$ and $\operatorname{rk}(w)<\beta$, the elements of $x$ and those of $w$ would be $\approx_{\beta}$-equivalent. Suppose $x \in \widehat{\mathcal{L}}^{(\beta)}$. Then $z=f_{\delta}(y)$, where $y$ is the $\approx_{\delta^{-}}$equivalence class in $\widehat{\mathcal{L}}^{(\delta)}$ which contains $x$. It follows that for all $\delta$ with $\beta \leq \delta<\alpha, \widehat{\mathcal{L}}^{(\delta)}$ is infinite, and hence $\operatorname{rk}(\widehat{\mathcal{L}})=\alpha$, proving our claim. Therefore, $\operatorname{rk}\left(\widehat{\mathcal{L}}^{(\beta)}\right)=\gamma$.

Let $\mathcal{B}_{0}, \ldots, \mathcal{B}_{k}$ be the minimal linear orderings of rank $\beta$. So, for each $x \in \widehat{\mathcal{L}}^{(\beta)}$, there is an $i_{x} \leq k$ such that $\mathcal{B}_{i_{x}} \preccurlyeq x$. By the previous lemma, there is an $i \leq k$ such that $\widehat{\mathcal{C}}=\left\{x \in \widehat{\mathcal{L}}^{(\beta)}: i_{x}=i\right\}$ has rank $\gamma$. Let $\mathcal{C} \preccurlyeq \widehat{\mathcal{C}}$ be an indecomposable of rank $\gamma$. Note that $\mathcal{B}=\mathcal{B}_{i}$ and $\mathcal{C}$ are as desired. 
Lemma 3.8. Let $\alpha>1$ be an indecomposable ordinal, and let $\mathcal{L} \in \mathbb{H}$. Then $\alpha \leq \operatorname{rk}(\mathcal{L})$ if and only if $\operatorname{lin}\left(\left\langle\alpha, \varepsilon_{0}, \varepsilon_{1}\right\rangle\right) \preccurlyeq \mathcal{L}$ for some $\varepsilon_{0}, \varepsilon_{1} \in\{+,-\}$.

Proof. The "if" part follows from the fact that $\operatorname{rk}\left(\operatorname{lin}\left(\left\langle\alpha, \varepsilon_{0}, \varepsilon_{1}\right\rangle\right)\right)=\alpha$.

Assume, without loss of generality, that $\mathcal{L}$ is indecomposable and has rank $\alpha$. So $|\tau(\mathcal{L})|=\operatorname{cf}(\alpha)$. Write $\mathcal{L}$ as $\sum_{\gamma \in \tau(\mathcal{L})} \mathcal{L}_{\gamma}$ and let $\varepsilon_{1}=\varepsilon_{\mathcal{L}}$.

To prove the lemma it will be enough to show that for every $\beta<\alpha$ there exists $\gamma$ such that either $\omega^{\beta}$ or $\omega^{\beta *}$ embeds in $\mathcal{L}_{\gamma}$, because then there exists $\varepsilon_{0} \in\{-,+\}$ such that for every $\beta<\alpha$ there is a $\gamma \in \tau(\mathcal{L})$ such that $\omega^{\beta \varepsilon_{0}} \preccurlyeq \mathcal{L}_{\gamma}$, and hence, by Lemma 1.10, $\operatorname{lin}\left(\left\langle\alpha, \varepsilon_{0}, \varepsilon_{1}\right\rangle\right) \preccurlyeq \mathcal{L}$. Assume first that $\alpha=\omega^{\delta}$ and $\delta$ is a limit ordinal. Then $\forall \beta<\alpha \exists \delta_{0}<\delta\left(\beta<\omega^{\delta_{0}}\right)$, and so, by inductive hypothesis, for some $\varepsilon^{0}$ and $\varepsilon^{1}, \operatorname{lin}\left(\left\langle\omega^{\delta_{0}}, \varepsilon^{0}, \varepsilon^{1}\right\rangle\right) \preccurlyeq \mathcal{L}$, and hence $\beta^{\varepsilon^{0}} \preccurlyeq \mathcal{L}$.

Assume now that the lemma is true for $\omega^{\delta}$ and let us prove it for $\alpha=$ $\omega^{\delta+1}$. Consider $\beta<\alpha$. For some $n<\omega, \beta<\omega^{\delta} \cdot n$. We need to prove that either $\omega^{\omega^{\delta} \cdot n}$ or $\omega^{\omega^{\delta} \cdot n *}$ embeds in $\mathcal{L}$. By the previous lemma there exist $\mathcal{A}_{1}, \ldots, \mathcal{A}_{2 n}$ of rank $\omega^{\delta} \cdot 2$ such that $\mathcal{A}_{1} \cdots \mathcal{A}_{2 n} \preccurlyeq \mathcal{L}$. We now claim that for each $i \leq 2 n$, either $\omega^{\omega^{\delta}}$ or $\omega^{\omega^{\delta} *}$ embeds in $\mathcal{A}_{i}$. Note that by an application of the pigeon-hole principle, this claim implies that either $\omega^{\omega^{\delta} \cdot n} \preccurlyeq \mathcal{L}$ or $\omega^{\omega^{\delta} \cdot n *} \preccurlyeq \mathcal{L}$.

By the previous lemma, there exist $\mathcal{B}_{0}$ and $\mathcal{B}_{1}$, both of rank $\omega^{\delta}$, such that $\mathcal{B}_{0} \cdot \mathcal{B}_{1} \preccurlyeq \mathcal{A}_{i}$. So, by the inductive hypothesis, there exist $\varepsilon^{0}, \varepsilon^{1}, \varepsilon^{2}, \varepsilon^{3} \in$ $\{+,-\}$ such that $\operatorname{lin}\left(\left\langle\omega^{\delta}, \varepsilon^{0}, \varepsilon^{1}\right\rangle\right) \preccurlyeq \mathcal{B}_{0}$ and $\operatorname{lin}\left(\left\langle\omega^{\delta}, \varepsilon^{2}, \varepsilon^{3}\right\rangle\right) \preccurlyeq \mathcal{B}_{1}$. If $\operatorname{cf}\left(\omega^{\delta}\right)$ $=\omega^{\delta}$, then $\omega^{\omega^{\delta} \varepsilon^{1}} \preccurlyeq \operatorname{lin}\left(\left\langle\omega^{\delta}, \varepsilon^{0}, \varepsilon^{1}\right\rangle\right) \preccurlyeq \mathcal{B}_{0} \preccurlyeq \mathcal{A}_{i}$. So, assume that $\operatorname{cf}\left(\omega^{\delta}\right)<\omega^{\delta}$. If $\varepsilon^{2}=\varepsilon^{3}$, then $\omega^{\omega^{\delta} \varepsilon^{2}} \preccurlyeq \mathcal{B}_{1} \preccurlyeq \mathcal{A}_{i}$ as desired. Otherwise, either $\varepsilon^{0}=\varepsilon^{2}$ or $\varepsilon^{0}=\varepsilon^{3}$. In any case, since $\operatorname{cf}\left(\omega^{\delta}\right) \preccurlyeq \operatorname{lin}\left(\left\langle\omega^{\delta}, \varepsilon^{2}, \varepsilon^{3}\right\rangle\right)$,

$$
\omega^{\omega^{\delta} \varepsilon^{0}}=\operatorname{lin}\left(\left\langle\omega^{\delta}, \varepsilon^{0}, \varepsilon^{0}\right\rangle\right) \preccurlyeq \operatorname{lin}\left(\left\langle\omega^{\delta}, \varepsilon^{0}, \varepsilon^{1}\right\rangle\right) \cdot \operatorname{lin}\left(\left\langle\omega^{\delta}, \varepsilon^{2}, \varepsilon^{3}\right\rangle\right) \preccurlyeq \mathcal{B}_{0} \cdot \mathcal{B}_{1} \preccurlyeq \mathcal{A}_{i}
$$

as required.

Proposition 3.5 follows easily from the previous two lemmas. For the case $\delta=1$ use the fact that if $\operatorname{rk}(\mathcal{L}) \geq 1$, then either $\omega \preccurlyeq \mathcal{L}$ or $\omega^{*} \preccurlyeq \mathcal{L}$.

The set $\{\operatorname{lin}(\vec{a}): \vec{a} \in$ F.1.o. $\delta$ is not exactly the set of minimal linear orderings of rank $\delta$, but a superset of it. The problem is that there might be $\vec{a}, \vec{b} \in$ F.1.o. $\delta$ such that $\operatorname{lin}(\vec{a}) \prec \operatorname{lin}(\vec{b})$. For example, if $\kappa>\omega$ is a regular cardinal, then

$$
\operatorname{lin}(\langle\kappa,+,+\rangle)=\kappa \prec \operatorname{lin}(\langle\kappa,-,+\rangle) .
$$

Also, if $\beta \geq \operatorname{cf}(\alpha) \cdot \omega$, then

$$
\operatorname{lin}(\langle\langle\alpha,+,+\rangle,\langle\beta,+,+\rangle\rangle) \prec \operatorname{lin}(\langle\langle\alpha,+,-\rangle,\langle\beta,+,+\rangle\rangle) .
$$

The reason is that, $\operatorname{since} \operatorname{lin}(\langle\alpha,+,+\rangle) \prec \operatorname{lin}(\langle\alpha,+,-\rangle) \cdot \operatorname{cf}(\alpha)$, we have

$$
\begin{aligned}
\operatorname{lin}(\langle\langle\alpha,+,+\rangle & ,\langle\beta,+,+\rangle\rangle)=\operatorname{lin}(\langle\alpha,+,+\rangle) \cdot \omega^{\beta} \\
\prec & \operatorname{lin}(\langle\alpha,+,-\rangle) \cdot \operatorname{cf}(\alpha) \cdot \omega^{\beta} \preccurlyeq \operatorname{lin}(\langle\alpha,+,-\rangle) \cdot \omega^{\operatorname{cf}(\alpha)+\beta} \\
\preccurlyeq & \operatorname{lin}(\langle\alpha,+,-\rangle) \cdot \omega^{\beta}=\operatorname{lin}(\langle\langle\alpha,+,-\rangle,\langle\beta,+,+\rangle\rangle) .
\end{aligned}
$$


Definition 3.9. Let $\delta$ be an ordinal with Cantor normal form $\delta=$ $\omega^{\alpha_{0}}+\cdots+\omega^{\alpha_{k}}$ where $\alpha_{0} \geq \alpha_{1} \geq \cdots \geq \alpha_{k}$. Let $\mathbb{F}_{\delta}$ be the set consisting of $\operatorname{lin}(\vec{a})$ for $\vec{a}=\left\langle a_{0}, \ldots, a_{k}\right\rangle \in$ F.1.o. such that for each $i$,

(1) if $\omega^{\alpha_{i}}$ is regular and $a_{i}=\left\langle\omega^{\alpha_{i}}, \varepsilon_{i, 0}, \varepsilon_{i, 1}\right\rangle$, then $\varepsilon_{i, 0}=\varepsilon_{i, 1}$,

(2) if $\left.\omega^{\alpha_{i+1}}\right\rangle \operatorname{cf}\left(\omega^{\alpha_{i}}\right)$ and $a_{i+1}=\left\langle\omega^{\alpha_{i+1}}, \varepsilon_{i+1,0}, \varepsilon_{i+1,1}\right\rangle$, then $\varepsilon_{a_{i}}=\varepsilon_{i+1,0}$.

COROLlary 3.10. For each ordinal $\delta, \mathbb{F}_{\delta}$ is the set of minimal linear orderings of rank $\delta$.

Proof. From the remark before Definition 3.9, it follows that for every $\vec{a} \in$ F.1.o. $\delta$, either $\operatorname{lin}(\vec{a}) \in \mathbb{F}_{\delta}$, or some element of $\mathbb{F}_{\delta}$ embeds in $\operatorname{lin}(\vec{a})$. So, all we need to prove is that $\mathbb{F}_{\delta} \subset \mathbb{H}$ is an antichain. Let $\delta=\omega^{\alpha_{0}}+\cdots+\omega^{\alpha_{k}}$ be in Cantor normal form as in the definition above. By induction on $k$ we show that if $\vec{a}, \vec{b} \in$ F.1.0. $\delta$ are such that

$$
\operatorname{lin}(\vec{a}) \in \operatorname{ide}\left(\vec{b} \frown\left\langle\omega^{\alpha_{k}}, \varepsilon_{\vec{b}}\right\rangle\right)
$$

(see Definition 3.11 below), then $\vec{a}=\vec{b}$. Suppose that $\vec{a}=\vec{c} \frown\left\langle\alpha_{k}, \varepsilon_{\vec{a}}^{0}, \varepsilon_{\vec{a}}\right\rangle$ and $\vec{b}=\vec{d}^{\wedge}\left\langle\alpha_{k}, \varepsilon_{\vec{b}}^{0}, \varepsilon_{\vec{b}}\right\rangle$, and that $\operatorname{lin}(\vec{a}) \preccurlyeq \operatorname{lin}(\vec{b}) \cdot \omega^{\beta \varepsilon_{\vec{b}}}$ for some $\beta<\omega^{\alpha_{k}}$.

If $\varepsilon_{\vec{a}} \neq \varepsilon_{\vec{b}}$, then $\operatorname{lin}(\vec{a}) \in \operatorname{ide}\left(\vec{d}^{\wedge}\left\langle\alpha_{k}, \varepsilon_{\vec{b}}^{0}\right\rangle\right)$, which is impossible because $\operatorname{rk}(\vec{a})=\operatorname{rk}\left(\vec{d}^{\frown}\left\langle\alpha_{k}, \varepsilon_{\vec{b}}^{0}\right\rangle\right)$. So $\varepsilon_{\vec{a}}=\varepsilon_{\vec{b}}$.

If $\omega^{\alpha_{k}}$ is regular, then $\varepsilon_{\vec{a}}^{0}=\varepsilon_{\vec{a}}=\varepsilon_{\vec{b}}^{0}$. If not, assume that $\beta>\operatorname{cf}\left(\omega^{\alpha_{k}}\right)$ is indecomposable, and hence $\operatorname{cf}\left(\omega^{\alpha_{k}}\right)+\beta=\beta<\beta+\operatorname{cf}\left(\omega^{\alpha_{k}}\right)$. Then, since

$$
\omega^{\beta \varepsilon_{\vec{a}}^{0}} \cdot \operatorname{cf}\left(\omega^{\alpha_{k}}\right)^{\varepsilon_{\vec{a}}} \preccurlyeq \operatorname{lin}\left(\omega^{\alpha_{k}}, \varepsilon_{\vec{a}}^{0}, \varepsilon_{\vec{a}}\right) \preccurlyeq \operatorname{lin}\left(\omega^{\alpha_{k}}, \varepsilon_{\vec{b}}^{0}, \varepsilon_{\vec{a}}\right) \cdot \omega^{\beta \varepsilon_{\vec{a}}},
$$

we have $\varepsilon_{\vec{a}}^{0}=\varepsilon_{\vec{b}}^{0}$, because otherwise $\varepsilon_{\vec{a}}^{0}=\varepsilon_{\vec{a}}$ and $\omega^{\beta+\operatorname{cf}\left(\omega^{\alpha_{\kappa}}\right)} \preccurlyeq \omega^{\operatorname{cf}\left(\omega^{\alpha_{k}}\right)+\beta}$, which is impossible.

What is left is to prove that $\vec{c}=\vec{d}$. Since $\operatorname{lin}\left(\left\langle\alpha_{k}, \varepsilon_{\vec{a}}^{0}, \varepsilon_{\vec{a}}\right\rangle\right) \npreceq \omega^{\beta \varepsilon_{\vec{b}}}$, we have $1+\operatorname{lin}(\vec{c})+1 \prec \operatorname{lin}\left(\vec{d}^{\wedge}\left\langle\alpha_{k}, \varepsilon_{\vec{a}}^{0}, \varepsilon_{\vec{a}}\right\rangle\right)$. If $\varepsilon_{\vec{a}}^{0}=\varepsilon_{\vec{a}}$, then $\operatorname{lin}(\vec{c}) \in \operatorname{ide}\left(\vec{d} \wedge\left\langle\alpha_{k}, \varepsilon_{\vec{a}}^{0}\right\rangle\right)$. If not, then necessarily $\operatorname{ide}\left(\vec{c}^{\curlyvee}\left\langle\alpha_{k}, \varepsilon_{\vec{a}}^{0}\right\rangle\right) \subseteq \operatorname{ide}\left(\vec{d}^{\wedge}\left\langle\alpha_{k}, \varepsilon_{\vec{a}}^{0}\right\rangle\right)$, so, we also have

$$
\operatorname{lin}(\vec{c}) \in \operatorname{ide}\left(\vec{d}^{\wedge}\left\langle\alpha_{k}, \varepsilon_{\vec{a}}^{0}\right\rangle\right) \subseteq \operatorname{ide}\left(\vec{d}^{\wedge}\left\langle\alpha_{k-1}, \varepsilon_{\vec{a}}^{0}\right\rangle\right) .
$$

If $\operatorname{cf}(\vec{c})=\operatorname{cf}\left(\omega^{\alpha_{k-1}}\right) \nless \omega^{\alpha_{k}}$, then actually $\operatorname{lin}(\vec{c}) \preccurlyeq \operatorname{lin}(\vec{d})$, and hence, by inductive hypothesis, $\vec{c}=\vec{d}$. Otherwise, $\varepsilon_{\vec{c}}=\varepsilon_{\vec{a}}^{0}=\varepsilon_{\vec{b}}^{0}=\varepsilon_{\vec{d}}$. So, again by inductive hypothesis, $\vec{c}=\vec{d}$.

As important as minimal linear orderings of a certain rank are minimal ideals. We will use minimal ideals to identify the ideals of $\mathcal{T}_{r}$ which have a certain rank. As we did in the previous subsection for linear orderings, we will define a class of ideals called the finitely alternating ideals. We will define a set F.id. of codes for finitely alternating ideals and an operation ide $(\cdot)$ that assigns an ideal to each member of F.id.: 
Definition 3.11. Given an indecomposable ordinal $\alpha>1$, let

$$
\operatorname{ide}(\langle\alpha, \varepsilon\rangle)=\mathbb{I}\left(\omega^{\alpha \varepsilon}\right)=\left\{\omega^{\beta \varepsilon}: \beta<\alpha\right\} .
$$

We call these ideals basic ideals. Let b.id. $=\{\langle\alpha, \varepsilon\rangle: \alpha \in \mathcal{O} n, \alpha$ indecomposable $\& \alpha>1\}$. Given a finitely alternating linear ordering $\mathcal{L}$ and a basic ideal $\mathbb{I}$ we let

$$
\mathcal{L} \cdot \mathbb{I}=\{\mathcal{A} \in \mathbb{H}: \exists \mathcal{B} \in \mathbb{I}(\mathcal{A} \preccurlyeq \mathcal{L} \cdot \mathcal{B})\} .
$$

Ideals of this form are called finitely alternating ideals. Let

$$
\text { F.id. }=\{\vec{a}\urcorner b: \vec{a} \in \text { F.1.o., } b \in \text { b.id. }\} .
$$

Given $\vec{b}=\vec{a}\urcorner b \in$ F.id., let $\operatorname{ide}(\vec{b})=\operatorname{lin}(\vec{a}) \cdot \operatorname{ide}(b)$.

Note that $\operatorname{lin}\left(\left\langle\alpha, \varepsilon_{0}, \varepsilon_{1}\right\rangle\right)=\operatorname{ide}\left(\left\langle\alpha, \varepsilon_{0}\right\rangle\right) \cdot \operatorname{cf}(\alpha)^{\varepsilon_{1}}$. Now, for each limit ordinal $\delta$, we want to define a finite set of finitely alternating ideals which contains all the minimal ideals of rank $\delta$.

Definition 3.12. Let $\delta$ have Cantor normal form $\delta=\omega^{\alpha_{0}}+\cdots+\omega^{\alpha_{k}}$ with $\alpha_{0} \geq \alpha_{1} \geq \cdots \geq \alpha_{k}>1$. Let

$$
\text { F.id. } \delta=\left\{\left\langle a_{0}, \ldots, a_{k-1}, a_{k}\right\rangle \in \text { F.id. }: \forall i \leq k\left(\operatorname{rk}\left(a_{i}\right)=\omega^{\alpha_{i}}\right)\right\} .
$$

Proposition 3.13. Let $\alpha$ be a limit ordinal and $\mathbb{I} \subseteq \mathbb{H}$ be an ideal. Then

$$
\alpha \leq \operatorname{rk}(\mathbb{I}) \Leftrightarrow(\exists \vec{b} \in \text { F.id. } \alpha) \operatorname{ide}(\vec{b}) \subseteq \mathbb{I} .
$$

Proof. The implication from right to left follows from the fact that for each $\vec{b} \in$ F.id. $\alpha, \operatorname{rk}(\operatorname{ide}(\vec{b}))=\alpha$.

Let us now prove the other direction, so assume that $\operatorname{rk}(\mathbb{I}) \geq \alpha$. Write $\alpha$ in Cantor's normal form and let $\omega^{\beta}$ be the last term. So $\alpha=\alpha_{0}+\omega^{\beta}$, where $\omega^{\beta}$ divides $\alpha_{0}$. Let $\left\{\mathcal{L}_{\gamma}: \gamma<\kappa\right\}$ be a cofinal sequence in $\mathbb{I}$. Assume that for all $\gamma<\kappa, \operatorname{rk}\left(\mathcal{L}_{\gamma}\right)>\alpha_{0}$, and let $\beta_{\gamma}$ be such that $\operatorname{rk}\left(\mathcal{L}_{\gamma}\right)=\alpha_{0}+\beta_{\gamma}$. Note that $\sup \left\{\beta_{\gamma}: \gamma<\kappa\right\}=\omega^{\beta}$. For each $\gamma$ there exist $\mathcal{A}_{\gamma}$ and $\mathcal{B}_{\gamma}$ of ranks $\alpha_{0}$ and $\beta_{\gamma}$ such that $\mathcal{A}_{\gamma} \cdot \mathcal{B}_{\gamma} \preccurlyeq \mathcal{L}_{\gamma}$. We can assume that each $\mathcal{A}_{\gamma}$ is a minimal linear ordering of rank $\alpha_{0}$ and hence a finitely alternating one. There exists $\vec{a} \in$ F.1.o. $\alpha_{0}$ such that $\left\{\beta_{\gamma}: \gamma<\kappa, \mathcal{A}_{\gamma}=\operatorname{lin}(\vec{a})\right\}$ is unbounded in $\omega^{\beta}$. Let $A=\left\{\gamma<\kappa: \mathcal{A}_{\gamma}=\operatorname{lin}(\vec{a})\right\}$. By transfinite induction and using the same idea as in Lemma 3.8, we can prove that for each $\delta<\omega^{\beta}$ there exists $\gamma \in A$ such that either $\omega^{\delta}$ or $\omega^{\delta *}$ embeds in $\mathcal{B}_{\gamma}$. So, for $\varepsilon$ either + or - , we have $\operatorname{ide}(\vec{a} \frown\langle\beta, \varepsilon\rangle)=\operatorname{lin}(\vec{a}) \cdot \operatorname{ide}\left(\omega^{\beta}, \varepsilon\right) \subseteq \mathbb{I}$.

The set $\mathbb{F}_{\delta}$ of minimal ideals of $\mathbb{H}$ of rank $\delta$ can be defined from F.id. $\delta$ using the ideas of Corollary 3.10 .

4. Examples of invariants. For each $\vec{a} \in$ F.1.o. $\cup$ F.id. we define a finite set $\operatorname{com}(\vec{a}) \subset$ F.l.o. which is the complement of $\vec{a}$ in the sense of Proposition 4.2 below. We will use these sets to compute the invariants of some finitely alternating linear orderings. Then we will use these invariants 
to define an algorithm that checks whether condition (3) of Proposition 2.14 holds.

Definition 4.1. First, let $\operatorname{com}(\langle\rangle)=\{\langle 1,+\rangle,\langle 1,-\rangle\}$. Second, consider $\vec{a} \in$ F.1.o. If $\vec{a}=\vec{d}^{\wedge}\left\langle\beta, \varepsilon_{0}, \varepsilon_{1}\right\rangle$ with $\beta>1$, let $\tau=\tau\left(\left\langle\beta, \varepsilon_{0}, \varepsilon_{1}\right\rangle\right)$ and let

$$
\begin{aligned}
\operatorname{com}(\vec{a})= & \left\{\vec{c}: \vec{c} \in \operatorname{com}\left(\vec{d}^{\wedge}\left\langle\beta, \varepsilon_{0}\right\rangle\right) \& \tau(\vec{c}) \npreceq \tau\right\} . \\
& \cup\left\{\vec{c}^{\wedge}\langle 1,+\rangle, \vec{c}^{\wedge}\langle 1,-\rangle: \vec{c} \in \operatorname{com}\left(\vec{d} \wedge\left\langle\beta, \varepsilon_{0}\right\rangle\right) \& \tau(\vec{c}) \preccurlyeq \tau\right\} .
\end{aligned}
$$

Otherwise, $\vec{a}=\vec{d}^{\wedge}\langle 1, \varepsilon\rangle$. Let $\tau=\tau(\langle 1, \varepsilon\rangle)$ and let

$$
\begin{aligned}
\operatorname{com}(\vec{a})= & \{\vec{c}: \vec{c} \in \operatorname{com}(\vec{d}) \& \tau(\vec{c}) \npreceq \tau\} \\
& \cup\{\vec{c} \frown\langle 1,+\rangle, \vec{c} \frown\langle 1,-\rangle: \vec{c} \in \operatorname{com}(\vec{d}) \& \tau(\vec{c}) \preccurlyeq \tau\} .
\end{aligned}
$$

Third, consider $\vec{b} \in$ F.id. and write $\vec{b}$ as $\vec{a} \wedge\langle\alpha, \varepsilon\rangle$, where $\vec{a} \in$ F.l.o. might be empty. Let $\mathbb{I}=\operatorname{ide}(\langle\alpha, \varepsilon\rangle)$, let $\bar{\epsilon}$ be the opposite of $\varepsilon$ (i.e., $\bar{\mp}=-$ and $-=+$ ), and let

$$
\begin{aligned}
\operatorname{com}(\vec{b})= & \{\vec{c}: \vec{c} \in \operatorname{com}(\vec{a}) \& \tau(\vec{c}) \notin \mathbb{I}\} \\
& \cup\{\vec{c} \frown\langle\alpha, \varepsilon, \varepsilon\rangle, \vec{c} \wedge\langle 1, \bar{\epsilon}\rangle: \vec{c} \in \operatorname{com}(\vec{a}) \& \tau(\vec{c}) \in \mathbb{I}\} .
\end{aligned}
$$

The definition above might look complicated at first. The motivation for it is just to make the following proposition work. Note that if $\vec{a} \in$ F.1.o. $U$ F.id. and $\vec{c} \in \operatorname{com}(\vec{a})$, then $\operatorname{lin}(\vec{c})$ is a product of linear orderings of the form $\omega^{\gamma}$ or $\omega^{\gamma *}$.

Proposition 4.2. Let $\vec{a} \in$ F.1.o., $\vec{b} \in$ F.id. and $\mathcal{L} \in \mathbb{H}$. Then

(1) $\mathcal{L} \npreceq \operatorname{lin}(\vec{a})$ if and only if $(\exists \vec{c} \in \operatorname{com}(\vec{a})) \operatorname{lin}(\vec{c}) \preccurlyeq \mathcal{L}$,

(2) $\mathcal{L} \notin \operatorname{ide}(\vec{b})$ if and only if $(\exists \vec{c} \in \operatorname{com}(\vec{b})) \operatorname{lin}(\vec{c}) \preccurlyeq \mathcal{L}$.

We start by proving the implications from right to left.

LEMMA 4.3.

(1) For every $\vec{b} \in$ F.1.o. and $\vec{c} \in \operatorname{com}(\vec{b}), \operatorname{lin}(\vec{c}) \npreceq \operatorname{lin}(\vec{b})$.

(2) For every $\vec{b} \in$ F.id. and $\vec{c} \in \operatorname{com}(\vec{b}), \operatorname{lin}(\vec{c}) \notin \operatorname{ide}(\vec{b})$.

Proof. We only show the second part, since the idea to prove the first one is very similar. We use induction on the size of $\vec{b}$. Suppose $\vec{b}=\vec{a} \frown\langle\alpha,+\rangle$, where $\vec{a} \in$ F.1.o., and consider $\vec{d} \in \operatorname{com}(\vec{b})$. Of course, the case $\vec{b}=\vec{a} \frown\langle\alpha,-\rangle$ is analogous. Assume, toward a contradiction, that $\operatorname{lin}(\vec{d}) \in \operatorname{ide}(\vec{b})$. So $\operatorname{lin}(\vec{d})$ $\preccurlyeq \operatorname{lin}(\vec{a}) \cdot \mathcal{C}$ for some $\mathcal{C} \in \operatorname{ide}(\langle\alpha,+\rangle)$. There are three possibilities for $\vec{d}$. The first one is that $\tau(\vec{d}) \notin \operatorname{ide}(\langle\alpha,+\rangle)$ and $\vec{d} \in \operatorname{com}(\vec{a})$. In this case, $\tau(\vec{d}) \npreceq \tau(\mathcal{C})$, and hence $\operatorname{lin}(\vec{d}) \preccurlyeq \operatorname{lin}(\vec{a})$, contrary to $\vec{d} \in \operatorname{com}(\vec{a})$. The second case is that $\vec{d}=\vec{c}^{\wedge}\langle 1,-\rangle$, where $\vec{c} \in \operatorname{com}(\vec{a})$ and $\tau(\vec{c}) \in \operatorname{ide}(\langle\alpha,+\rangle)$. In this case, since $\omega^{*} \npreceq \mathcal{C}$, necessarily $\operatorname{lin}(\vec{c}) \preccurlyeq \operatorname{lin}(\vec{a})$, again contradicting the choice of $\vec{c}$. The last case is that $\vec{d}=\vec{c} \frown\langle\alpha,+,+\rangle$, where $\vec{c} \in \operatorname{com}(\vec{a})$ and $\tau(\vec{c}) \in \operatorname{ide}(\langle\alpha,+\rangle)$. 
Let $\mathcal{C}_{1}$ be such that $\mathcal{C} \preccurlyeq \omega^{|\tau(\vec{c})|} \cdot \mathcal{C}_{1} \prec \omega^{\alpha}$. Then $\omega^{\alpha}=\omega^{\alpha-|\tau(\vec{c})|} \npreceq \mathcal{C}_{1}$. So, since $\operatorname{lin}(\vec{c}) \cdot \omega^{\alpha}=\operatorname{lin}(\vec{d}) \preccurlyeq \operatorname{lin}(\vec{a}) \cdot \mathcal{C} \preccurlyeq \operatorname{lin}(\vec{a}) \cdot \omega^{|\tau(\vec{c})|} \cdot \mathcal{C}_{1}$, we have $\operatorname{lin}(\vec{c})+1 \preccurlyeq \operatorname{lin}(\vec{a}) \cdot \omega^{|\tau(\vec{c})|}$. Then, by Lemma $1.8(3), \operatorname{lin}(\vec{c}) \preccurlyeq \operatorname{lin}(\vec{a})$, again contradicting the assumption that $\vec{c} \in \operatorname{com}(\vec{a})$.

Before proving the proposition we need a few definitions and observations about presentations of equimorphism types of indecomposable linear orderings.

Definition 4.4. An indecomposable linear ordering $\mathcal{L}$ is hereditarily normal if either

- $\mathcal{L}=1$, or

- $\mathcal{L}=\sum_{i \in \omega^{\varepsilon}} \mathcal{L}_{i}$, where each $\mathcal{L}_{i}$ is hereditarily normal and appears infinitely often in the sum, or

- $\mathcal{L}=\sum_{\gamma \in \kappa^{\varepsilon}} \mathcal{L}_{\gamma}$, where $\kappa>\omega$ is a regular cardinal, and for each $\alpha, \beta<\kappa$,

$$
\sum_{\gamma \in\left[\omega^{\alpha} \cdot \beta, \omega^{\alpha} \cdot(\beta+1)\right)^{\varepsilon}} \mathcal{L}_{\gamma}
$$

is a hereditarily normal indecomposable linear ordering.

Note that being hereditarily normal is a property of the isomorphism type of $\mathcal{L}$ and not of its equimorphism type.

Given a hereditarily normal indecomposable linear ordering $\mathcal{L}$, we let $\mathrm{bSeg}(\mathcal{L})$ be the set of building segments of $\mathcal{L}$. More precisely, $\mathrm{bSeg}(1)=\{1\}$; if $\mathcal{L}=\sum_{\omega^{\varepsilon}} \mathcal{L}_{i}$, then $\operatorname{bSeg}(\mathcal{L})=\{\mathcal{L}\} \cup \bigcup_{i \in \omega} \operatorname{bSeg}\left(\mathcal{L}_{i}\right)$; and if $\mathcal{L}=\sum_{\gamma \in \kappa^{\varepsilon}} \mathcal{L}_{\gamma}$, then

$$
\operatorname{bSeg}(\mathcal{L})=\{\mathcal{L}\} \cup \bigcup_{\alpha, \beta<\kappa} \operatorname{bSeg}\left(\sum_{\gamma \in\left[\omega^{\alpha} \cdot \beta, \omega^{\alpha} \cdot(\beta+1)\right)^{\varepsilon}} \mathcal{L}_{\gamma}\right) .
$$

Note that $\operatorname{bSeg}(\mathcal{L})$ is not a set of isomorphism types, but a set of subsets of $\mathcal{L}$.

Lemma 4.5. Every indecomposable linear ordering is equimorphic to a hereditarily normal one.

Proof. The proof is by transfinite induction. Let $\mathcal{L} \in \mathbb{H}$ be such that $\mathcal{L}=\sum_{\gamma \in \tau(\mathcal{L})} \mathcal{L}_{\gamma}$ and each $\mathcal{L}_{\gamma}$ is hereditarily normal. If $\tau(\mathcal{L})=\omega$, then since $\mathcal{L}$ is indecomposable,

$$
\sum_{\gamma \in \omega} \mathcal{L}_{\gamma} \sim \sum_{n \in \omega}\left(\sum_{\gamma<n} \mathcal{L}_{\gamma}\right)=\mathcal{L}_{0}+\mathcal{L}_{0}+\mathcal{L}_{1}+\mathcal{L}_{0}+\mathcal{L}_{1}+\mathcal{L}_{2}+\cdots,
$$

which is hereditarily normal. Suppose now that $\tau(\mathcal{L})=\kappa>\omega$. For each $\gamma<\kappa$, by transfinite recursion we define $\widehat{\mathcal{L}}_{\gamma} \in \mathbb{H}$ such that if $\delta<\gamma$, then $\widehat{\mathcal{L}}_{\delta}$ is an initial segment of $\widehat{\mathcal{L}}_{\gamma}$. Let $\widehat{\mathcal{L}}_{0}=\mathcal{L}_{0}$. If $\gamma=\delta+1$, let $\widehat{\mathcal{L}}_{\gamma}=\left(\widehat{\mathcal{L}}_{\delta}+\mathcal{L}_{\gamma}\right) \cdot \omega$. If $\gamma$ is a limit ordinal and $\operatorname{cf}(\gamma)=\omega$, let $\gamma_{0}<\gamma_{1}<\cdots$ be a cofinal sequence in $\gamma$ and let $\widehat{\mathcal{L}}_{\gamma}$ be an $\omega$-sum of $\left\{\mathcal{L}_{\gamma}, \mathcal{L}_{\gamma_{0}}, \mathcal{L}_{\gamma_{1}}, \ldots\right\}$ in which each term appears 
infinitely often. If $\operatorname{cf}(\gamma)>\omega$, let $\widehat{\mathcal{L}}_{\gamma}$ be the union of the $\widehat{\mathcal{L}}_{\delta}, \delta<\gamma$. Note that $\widehat{\mathcal{L}}_{\kappa}$ is hereditarily normal and equimorphic to $\mathcal{L}$.

OBSERVATION 4.6. Let $\mathcal{L}$ be a hereditarily normal indecomposable linear ordering.

(1) $\langle\operatorname{bSeg}(\mathcal{L}), \subseteq\rangle$ is well-founded, because $\langle\mathbb{H}, \preccurlyeq\rangle$ is well-founded, and if $\mathcal{A}, \mathcal{B} \in \operatorname{bSeg}(\mathcal{L})$ and $\mathcal{A} \subsetneq \mathcal{B}$, then $\mathcal{A} \prec \mathcal{B}$.

(2) Any two elements of $\operatorname{bSeg}(\mathcal{L})$ are either disjoint or one contains the other.

(3) Every $\mathcal{A} \in \operatorname{bSeg}(\mathcal{L}) \backslash\{\mathcal{L}\}$ has a successor, $\operatorname{succ}(\mathcal{A})$, in $\operatorname{bSeg}(\mathcal{L})$, that is, the least element of $\operatorname{bSeg}(\mathcal{L})$ that strictly includes $\mathcal{A}$. This is because $\{\mathcal{B} \in \operatorname{bSeg}(\mathcal{L}): \mathcal{A} \subsetneq \mathcal{B}\}$ is well-ordered. Moreover, the successor of $\mathcal{A}$ is either an $\omega$-sum or an $\omega^{*}$-sum of members of $\operatorname{bSeg}(\mathcal{L})$, infinitely many of which are isomorphic to $\mathcal{A}$.

(4) If $\mathcal{A}, \mathcal{B} \in \operatorname{bSeg}(\mathcal{L})$, there is a least $\mathcal{C} \in \operatorname{bSeg}(\mathcal{L})$ which contains both. Moreover, if $\mathcal{A}$ and $\mathcal{B}$ are incomparable, then $\mathcal{C}$ is a successor element. This is because if $\mathcal{C}$ is a $\tau$-sum and $|\tau|>\omega$, then there is a smaller building segment that contains both $\mathcal{A}$ and $\mathcal{B}$.

(5) Let $\mathbb{A}$ be an antichain of $\operatorname{bSeg}(\mathcal{L})$, and let $\widetilde{\mathbb{A}}$ be the upwards closure of $\mathbb{A}$. Define

$$
\mathbb{B}=\mathbb{A} \cup\{\mathcal{B} \in \operatorname{bSeg}(\mathcal{L}): \mathcal{B} \notin \widetilde{\mathbb{A}} \& \operatorname{succ}(\mathcal{B}) \in \widetilde{\mathbb{A}}\}
$$

We claim that $\mathbb{B}$ is a maximal antichain in $\operatorname{bSeg}(\mathcal{L})$. Suppose not, and let $\mathcal{C} \in \operatorname{bSeg}(\mathcal{L})$ be incomparable with all the elements of $\mathbb{B}$. Let $\mathcal{C}_{1} \in \widetilde{\mathbb{A}}$ be the least that contains $\mathcal{C}$. Then $\mathcal{C}_{1}$ is the least upper bound of $\mathcal{C}$ and some $\mathcal{B} \in \mathbb{A}$, so it is the successor of some $\mathcal{C}_{2} \supseteq \mathcal{C}$. But then $\mathcal{C}_{2} \in \mathbb{B}$, contradicting the definition of $\mathcal{C}$.

Proof of Proposition 4.2. The direction from right to left follows from Lemma 4.3.

Consider $\vec{a} \in$ F.1.o. and assume that $\mathcal{L} \npreceq \operatorname{lin}(\vec{a})$. We want to show that $\operatorname{lin}(\vec{c}) \preccurlyeq \mathcal{L}$ for some $\vec{c} \in \operatorname{com}(\vec{a})$. If $\vec{a}=\langle\rangle$, then $\mathcal{L}$ is infinite, and then either $\omega \preccurlyeq \mathcal{L}$ or $\omega^{*} \preccurlyeq \mathcal{L}$. So, suppose that $\vec{a}=\vec{d}^{\frown}\left\langle\alpha, \varepsilon_{0}, \varepsilon_{1}\right\rangle$ where $\alpha>1$. The case $\vec{a}=\vec{d}^{\frown}\langle 1, \varepsilon\rangle$ is similar, but simpler. Consider the set of building segments of $\mathcal{L}$ which do not belong to ide $\left(\vec{d} \frown\left\langle\alpha, \varepsilon_{0}\right\rangle\right)$. Suppose first that there is some $\widehat{\mathcal{L}} \in \operatorname{bSeg}(\mathcal{L}) \backslash\{\mathcal{L}\}$ and $\widehat{\mathcal{L}} \notin \operatorname{ide}\left(\vec{d}^{\frown}\left\langle\alpha, \varepsilon_{0}\right\rangle\right)$. Then there exists $\vec{c} \in \operatorname{com}\left(\vec{d} \frown\left\langle\alpha, \varepsilon_{0}\right\rangle\right)$ such that $\operatorname{lin}(\vec{c}) \preccurlyeq \widehat{\mathcal{L}}$. If $\tau(\vec{c}) \npreceq \tau\left(\left\langle\alpha, \varepsilon_{0}, \varepsilon_{1}\right\rangle\right)$, then $\vec{c} \in \operatorname{com}(\vec{a})$, and we are done. Otherwise, both $\vec{c} \frown\langle 1,+\rangle$ and $\vec{c} \frown\langle 1,-\rangle$ belong to $\operatorname{com}(\vec{a})$. Since either $\widehat{\mathcal{L}} \cdot \omega$ or $\widehat{\mathcal{L}} \cdot \omega^{*}$ embeds in $\mathcal{L}$, either $\operatorname{lin}(\vec{c} \frown\langle 1,+\rangle) \preccurlyeq \mathcal{L}$ or $\operatorname{lin}(\vec{c} \frown\langle 1,-\rangle) \preccurlyeq \mathcal{L}$. Suppose now that $\mathcal{L}$ is the only member of $\operatorname{bSeg}(\mathcal{L})$ which is not in $\operatorname{ide}\left(\vec{d}^{\frown}\left\langle\alpha, \varepsilon_{0}\right\rangle\right)$. Let $\vec{c} \in \operatorname{com}\left(\vec{d}^{\frown}\left\langle\alpha, \varepsilon_{0}\right\rangle\right)$ be such that $\operatorname{lin}(\vec{c}) \preccurlyeq \mathcal{L}$. 
Note that $\tau(\vec{c})=\tau(\mathcal{L})$, because otherwise $\operatorname{lin}(\vec{c})$ would embed into a smaller building segment of $\mathcal{L}$. If $\tau(\vec{c}) \npreceq \tau\left(\left\langle\alpha, \varepsilon_{0}, \varepsilon_{1}\right\rangle\right)$, then $\vec{c} \in \operatorname{com}(\vec{a})$, and we are done. Otherwise, $\tau(\mathcal{L})=\tau(\vec{c}) \preccurlyeq \tau\left(\left\langle\alpha, \varepsilon_{0}, \varepsilon_{1}\right\rangle\right)$. But then we have $\mathcal{L} \preccurlyeq$ $\operatorname{ide}\left(\vec{d}^{\wedge}\left\langle\alpha, \varepsilon_{0}\right\rangle\right) \cdot \tau\left(\left\langle\alpha, \varepsilon_{0}, \varepsilon_{1}\right\rangle\right)=\operatorname{lin}(\vec{a})$, contradicting our initial assumptions.

Consider now $\vec{b}=\vec{d}^{\urcorner}\langle\alpha,+\rangle \in$ F.id. Let $\left\{\mathcal{L}_{i}: i \in \widehat{L}\right\} \subseteq \operatorname{bSeg}(\mathcal{L})$ be the set of minimal building segments of $\mathcal{L}$ which do not embed in $\operatorname{lin}(\vec{d})$. For each $i \in \widehat{L}$ there exists $\vec{c}_{i} \in \operatorname{com}(\vec{d})$ such that $\operatorname{lin}\left(\vec{c}_{i}\right) \preccurlyeq \mathcal{L}_{i}$. Note that for each $i \in \widehat{L}, \tau\left(\vec{c}_{i}\right)=\tau\left(\mathcal{L}_{i}\right)$, because otherwise $\operatorname{lin}(\vec{c})$ would embed into a smaller building segment of $\mathcal{L}_{i}$. If for some $i \in \widehat{L}, \tau\left(\vec{c}_{i}\right) \notin \operatorname{ide}(\langle\alpha,+\rangle)$, then $\vec{c}_{i} \in \operatorname{com}(\vec{a})$, so we are done. Suppose now that for every $i \in \widehat{L}$, $\tau\left(\mathcal{L}_{i}\right)=\tau\left(\vec{c}_{i}\right) \in \operatorname{ide}(\langle\alpha,+\rangle)$.

Extend $\left\{\mathcal{L}_{i}: i \in \widehat{L}\right\}$ to a maximal antichain $\left\{\mathcal{L}_{i}: i \in \bar{L}\right\} \subseteq \operatorname{bSeg}(\mathcal{L})$ as in Observation 4.6(5). For $i \in \widehat{L}$, let $\kappa_{i}=\tau\left(\mathcal{L}_{i}\right)$, and for $i \in \bar{L} \backslash \widehat{L}$, let $\kappa_{i}=1$. Let $\overline{\mathcal{L}}=\left\langle\bar{L}, \leq_{\overline{\mathcal{L}}}\right\rangle$, where $\leq_{\overline{\mathcal{L}}}$ is the ordering on $\bar{L}$ induced by the ordering on $\mathcal{L}$ in the obvious way. First, we observe that if some $\mathcal{A} \in$ $\operatorname{bSeg}(\mathcal{L})$ extending some element of $\overline{\mathcal{L}}$ is indecomposable to the left, then $\operatorname{lin}\left(\vec{c}_{i}\right) \cdot \omega^{*} \preccurlyeq \mathcal{L}$ for some $i$, and so we are done. So, we can assume that $\overline{\mathcal{L}}$ is an ordinal. If $\sum_{i \in \overline{\mathcal{L}}} \kappa_{i} \prec \omega^{\alpha}$ then we would have $\mathcal{L} \in \operatorname{ide}(\vec{b})$, contradicting our assumptions. So, $\omega^{\alpha} \preccurlyeq \sum_{i \in \overline{\mathcal{L}}} \kappa_{i}$. We note that $\sum_{i \in \overline{\mathcal{L}}} \kappa_{i}=\sum_{i \in \widehat{\mathcal{L}}} \kappa_{i}$. The reason is the following. Let $\mathcal{A} \in \operatorname{bSeg}(\mathcal{L})$ be a successor of some $\mathcal{L}_{i}, i \in \bar{L}$; it is also a successor of some $\mathcal{L}_{j}, j \in \widehat{L}$. So, $\mathcal{A}=\sum_{k \in \overline{\mathcal{B}}} \mathcal{L}_{k}$, where $\overline{\mathcal{B}} \subseteq \overline{\mathcal{L}}$ has order type either $\omega$ or $\omega^{*}$, and each $\mathcal{L}_{k}$ appears infinitely often in the sum.

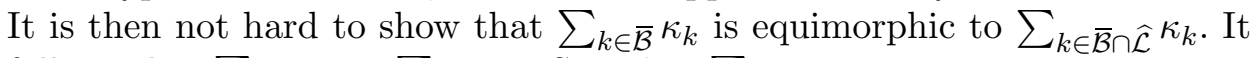
follows that $\sum_{i \in \overline{\mathcal{L}}} \kappa_{i}=\sum_{i \in \widehat{\mathcal{L}}} \kappa_{i}$. So, $\omega^{\alpha} \preccurlyeq \sum_{i \in \widehat{\mathcal{L}}} \kappa_{i}$.

It is known that $\omega^{\alpha}$ is strongly indecomposable or indivisible (see [Fra00, 6.8.1]). That is, for every coloring of $\omega^{\alpha}$ into finitely many colors, there is a monochromatic subset of $\omega^{\alpha}$ equimorphic to $\omega^{\alpha}$. Therefore, there is some $\vec{c} \in \operatorname{com}(\vec{d})$ such that $\omega^{\alpha} \preccurlyeq \sum_{i \in \widehat{\mathcal{L}}_{\vec{c}_{i}=\vec{c}}} \kappa_{i}=\tau(\vec{c}) \cdot\left\{i \in \widehat{\mathcal{L}}: \vec{c}_{i}=\vec{c}\right\}$. Since $\alpha$ is indecomposable and $\tau(\vec{c})<\alpha$, we have $\tau(\vec{c})+\alpha=\alpha$. Therefore $\omega^{\alpha} \preccurlyeq\left\{i \in \widehat{\mathcal{L}}: \vec{c}_{i}=\vec{c}\right\}$, and hence $\operatorname{lin}(\vec{c} \frown\langle\alpha,+,+\rangle)=\operatorname{lin}(\vec{c}) \cdot \omega^{\alpha} \preccurlyeq \mathcal{L}$.

Now we use the results above to compute the invariants of linear orderings which are products of indecomposable ordinals or reverse indecomposable ordinals. Note that if $\omega^{\delta}$ is an indecomposable ordinal, and $\delta=\omega^{\alpha_{0}}+\cdots+\omega^{\alpha_{k}}$, then

$$
\omega^{\delta}=\operatorname{lin}\left(\left\langle\omega^{\alpha_{0}},+,+\right\rangle, \ldots,\left\langle\omega^{\alpha_{k}},+,+\right\rangle\right) .
$$

Corollary 4.7. Let $\vec{a} \in$ F.1.o. be such that $\operatorname{lin}(\vec{a})$ is a product of indecomposable ordinals and reverse ordinals. Then

$$
\mathrm{T}(\operatorname{lin}(\vec{a}))=[\langle\operatorname{rk}(\vec{a}), \varepsilon\rangle ;\{\mathrm{T}(\operatorname{lin}(\vec{b})): \vec{b} \in \operatorname{com}(\vec{a}), \operatorname{rk}(\vec{b})<\operatorname{rk}(\vec{a})\}] .
$$


Proof. The proof is straightforward from the proposition above and the definition of $\mathrm{T}(\cdot)$.

ObServation 4.8. Suppose that $T_{0}, \ldots, T_{k} \in \mathcal{T}_{r}$. Let $\mathbb{I}=\{\operatorname{lin}(T): T \in$ $\left.\mathcal{I}_{T_{0}, \ldots, T_{k}}^{\alpha}\right\}$. We note that

$$
\operatorname{rk}\left(\mathcal{I}_{T_{0}, \ldots, T_{k}}^{\alpha}\right)=\alpha \Leftrightarrow \exists \vec{b} \in \text { F.id. }_{\alpha}(\operatorname{ide}(\vec{b}) \subseteq \mathbb{I}) .
$$

We can check whether ide $(\vec{b}) \subseteq \mathbb{I}$ because

$$
\operatorname{ide}(\vec{b}) \subseteq \mathbb{I} \Leftrightarrow \forall i \leq k \exists \vec{c} \in \operatorname{com}(\vec{b})\left(\mathrm{T}(\operatorname{lin}(\vec{c})) \preccurlyeq T_{i}\right) .
$$

5. Open questions. In this section we mention how to extend our results to the class of $\sigma$-scattered linear orderings, and some possible directions for future work.

Operations on $\mathcal{T}_{r}$. The main question we leave open is whether, given a tree with labels in $\mathcal{O} n \times\{+,-\}$, we can tell if it belongs to $\mathcal{T}_{r}$ via a finite manipulation of the symbols in the tree, using some basic operations on ordinals. Using our results, it remains to find a procedure to check that an ideal in $\mathcal{T}_{r}$ has a certain cofinality, the ideal being given by the minimal elements of its complement (see Proposition 2.14).

Then comes the question of which operations on $\mathcal{T}_{r}$ can be done via a finite manipulation of symbols. An interesting operation is the product of linear orderings.

Another possible definition. A variant of the definition of the invariant $\mathrm{T}(\mathcal{L})$ could be the following. Instead of using $\mathbb{I}_{\mathcal{L}}$, we can take $\mathbb{I}_{\mathcal{L}}^{\mathrm{min}}$, the minimal ideal such that $\mathcal{L}=\mathbb{I}_{\mathcal{L}}^{\min } \cdot \tau(\mathcal{L})$. Here is a proof that such a minimal ideal exists.

Lemma 5.1. Let $\mathcal{L} \in \mathbb{H}$. There exists an ideal $\mathbb{J} \subset \mathbb{H}_{\mathrm{rk}(\mathcal{L})}$ which is the least one such that $\mathbb{J} \cdot \tau(\mathcal{L})=\mathcal{L}$.

Proof. As mentioned in the introduction, since $\mathbb{H}$ is a better-quasiordering, the class of ideals of $\mathbb{H}$ is well-quasiordered by inclusion. Let $\mathbb{I}_{0}, \ldots, \mathbb{I}_{k-1}$ be the set of minimal ideals $\mathbb{I}$ such that $\mathbb{I} \cdot \tau(\mathcal{L})=\mathcal{L}$. We claim that $k=1$. Suppose not, and let $\mathbb{I}=\mathbb{I}_{0} \cap \mathbb{I}_{1}$. Let $\mathcal{A} \in \mathbb{I}_{0}$. If $\tau(\mathcal{A}) \nprec \tau(\mathcal{L})$, then $\mathcal{A}$ embeds into one of the summands of $\mathbb{I}_{1} \cdot \tau(\mathcal{L})=\mathcal{L}$, and hence $\mathcal{A} \in \mathbb{I}_{0} \cap \mathbb{I}_{1}=\mathbb{I}$. Therefore, every $\mathcal{A} \in \mathbb{I}_{0} \backslash \mathbb{I}$ has $\tau(\mathcal{A}) \prec \tau(\mathcal{L})$. We claim that for each $\mathcal{A} \in \mathbb{I}_{0} \backslash \mathbb{I}$, there exists an $\alpha_{\mathcal{A}} \prec \tau(\mathcal{L})$ and a set $\left\{\mathcal{B}_{i}: i<\alpha_{\mathcal{A}}\right\} \subseteq \mathbb{I}$ such that $\mathcal{A} \preccurlyeq \sum_{i \in \alpha_{\mathcal{A}}} \mathcal{B}_{i}$. The proof is by induction on the rank of $\mathcal{A}$. Since $\tau(\mathcal{A}) \prec \tau(\mathcal{L}), \mathcal{A}=\sum_{j \in \tau(\mathcal{A})} \mathcal{A}_{j}$ where each $\mathcal{A}_{j} \in \mathbb{I}_{0}$ has rank smaller than $\mathcal{A}$. So, for each $\mathcal{A}_{j}$ there exists $\alpha_{\mathcal{A}_{j}}<\tau(\mathcal{L})$ and a set $\left\{\mathcal{B}_{j, k}: k<\alpha_{\mathcal{A}_{j}}\right\} \subseteq \mathbb{I}$ such 
that $\mathcal{A}_{j} \preccurlyeq \sum_{k \in \alpha_{\mathcal{A}_{j}}} \mathcal{B}_{j, k}$. Therefore

$$
\mathcal{A} \preccurlyeq \sum_{j \in \tau(\mathcal{A})} \sum_{k \in \alpha_{j}} \mathcal{B}_{j, k} \preccurlyeq \sum_{\langle k, j\rangle \in \sum_{i \in \tau(\mathcal{A})} \alpha_{i}} \mathcal{B}_{j, k} .
$$

Let $\alpha_{\mathcal{A}}=\sum_{j \in \tau(\mathcal{A})} \alpha_{j}$. Since $|\tau(\mathcal{L})|$ is regular, $\alpha_{\mathcal{A}}<\tau(\mathcal{L})$.

Now, using Lemma 1.10 , we infer that $\mathbb{I}_{0} \cdot \tau(\mathcal{L}) \preccurlyeq \mathbb{I} \cdot \tau(\mathcal{L})$ and hence that $\mathcal{L}=\mathbb{I} \cdot \tau(\mathcal{L})$. This contradicts the minimality of $\mathbb{I}_{0}$ and $\mathbb{I}_{1}$.

Invariants for Galvin's class. The same idea we used to define invariants for $\mathbb{S}$ can be used to define equimorphism invariants for the class of $\sigma$ scattered linear orderings.

Definition 5.2. We say that $\mathcal{L}$ is $\sigma$-scattered if it is a countable union of scattered linear orderings.

This class was first studied by Galvin. The reason why one can define invariants for this class as we did for the class of scattered linear orderings is that versions of Theorems 1.1, 1.3 and 1.4 can be proved for this class. (Each of these theorems is due either to Galvin or to Laver; see [Lav71].) In this case, the labels of the trees should also include information about how the linear ordering is constructed from smaller ones. In other words, the label at the root of $\mathrm{T}(\mathcal{L})$ should include $\tau(\mathcal{L})$, which is now an element of $\mathcal{R} e g^{ \pm} \cup\left\{\eta_{\alpha, \beta}:\langle\alpha, \beta\rangle\right.$ is admissible $\}$.

\section{References}

[EH63] P. Erdős and A. Hajnal, On a classification of denumerable order types and an application to the partition calculus, Fund. Math. 51 (1962/1963), 117-129.

[Fra48] R. Fraïssé, Sur la comparaison des types d'ordres, C. R. Acad. Sci. Paris 226 (1948), 1330-1331.

[Fra00] - Theory of Relations, rev. ed., North-Holland, 2000.

[Hau08] F. Hausdorff, Grundzüge einer Theorie der geordneten Mengen, Math. Ann. 65 (1908), 435-505.

[Jul69] P. Jullien, Contribution à l'étude des types d'ordre dispersés, $\mathrm{PhD}$ thesis, Marseille, 1969.

[Kun80] K. Kunen, Set Theory. An Introduction to Independence Proofs, North-Holland, 1980.

[Lav71] R. Laver, On Fraïssé's order type conjecture, Ann. of Math. 93 (2) (1971), 89-111.

[Mona] A. Montalbán, Equivalence between Fraïssé's conjecture and Jullien's theorem, Ann. Pure Appl. Logic 139 (2006), 1-42.

[Monb] -, Indecomposable linear orderings and hyperarithmetic analysis, submitted.

[Mon05a] —, Beyond the arithmetic, PhD thesis, Cornell Univ., Ithaca, NY, 2005.

[Mon05b] - Up to equimorphism, hyperarithmetic is recursive, J. Symbolic Logic 70 (2005), 360-378. 
[NW68] C. St. J. A. Nash-Williams, On better-quasi-ordering transfinite sequences, Proc. Cambridge Philos. Soc. 64 (1968), 273-290.

[Ros82] J. Rosenstein, Linear Orderings, Academic Press, New York, 1982.

Department of Mathematics

University of Chicago

Chicago, IL 60637, U.S.A.

E-mail: antonio@math.uchicago.edu

URL: www.math.uchicago.edu/ antonio

Received 14 September 2005;

in revised form 18 April 2006 